\title{
Civilisations
}

Revue internationale d'anthropologie et de sciences

humaines

$52-2 \mid 2005$

Museums, collections, interpretations

\section{De la collection missionnaire au commerce équitable}

\section{Astrid de Hontheim}

\section{OpenEdition \\ Journals}

Édition électronique

URL : http://journals.openedition.org/civilisations/758

DOI : $10.4000 /$ civilisations.758

ISSN : 2032-0442

\section{Éditeur}

Institut de sociologie de l'Université Libre de Bruxelles

\section{Édition imprimée}

Date de publication : 1 juin 2005

Pagination : 75-104

ISSN : 0009-8140

\section{Référence électronique}

Astrid de Hontheim, "De la collection missionnaire au commerce équitable », Civilisations [En ligne], 52-2 | 2005, mis en ligne le 02 février 2009, consulté le 02 mai 2019. URL : http:// journals.openedition.org/civilisations/758; DOI : 10.4000/civilisations.758 


\title{
De la collection missionnaire au commerce équitable
}

\author{
Astrid de HONTHEIM
}

\section{Introduction}

En 1958, quatre missionnaires croisiers ${ }^{1}$ américains OSC furent envoyés en Asmat, dans les marais du centre sud de la Papouasie occidentale. Le premier missionnaire, le père G. Zegwaard MSC, y avait accosté en 1953. Comme leurs prédécesseurs, la perspective d'une mission difficile dans un endroit à la réputation désastreuse comme la NouvelleGuinée plaisait aux Croisiers. L'analyse de la correspondance montre qu'ils n'auraient pas été intéressés par des missions dans un endroit « facile» ou « plaisant» mais bien « primitif et rude comme la Nouvelle-Guinée» (Mischke, 1957). On retrouve cette attirance pour les terres « vierges » accompagnée d'une valorisation de la combativité missionnaire dans les interviews des religieux du Sacré-Cœur MSC, pour qui la NouvelleGuinée est le lieu d'accomplissement par excellence des « vrais » missionnaires. Seuls s'y rendaient les candidats dont la condition physique était jugée suffisante, « avec un corps solide ». Ainsi, la pratique difficile du terrain l'entourait tant de prestige que seule l'élite y avait accès. Nous allons voir qu'une fois sur place, les Croisiers se découvrirent d'autres motivations.

En 1961, Alphonse Sowada, le futur évêque des Croisiers (de 1969 à 2002), obtint après un an et demi son Master's en anthropologie à la Catholic University of America de Washington DC. C'est le premier Croisier à prendre cette orientation; d'autres lui emboîtèrent le pas. La personnalité d'Alphonse se révéla déterminante dans l'orientation du travail de mission de ses coreligionnaires. Au nom de leur formation anthropologique, les Croisiers s'efforcèrent de détruire le moins possible dans un souci déclaré de « préservation de la culture », principalement dans le domaine de l'art (de Hontheim, 2005). Cela ne date pas d'hier. L'histoire de l'ordre telle qu'elle est représentée sur les vitraux de l'église d'Onamia au Minnesota montre en effet qu'avant d'avoir cherché à préserver la culture, les Croisiers préservèrent le savoir par les copies de manuscrits et les enluminures.

1. Il s'agit de l'Ordre de la Sainte Croix, fondé en 1215 à Clairlieu en Belgique dans la continuité de la troisième croisade. Les Croisiers furent les principaux responsables de la christianisation des Asmat. 
Conformément à cet état d'esprit, les Croisiers furent les initiateurs d'un vaste mouvement de protection et de mise en valeur de la production matérielle asmat. Avec le concours de collectionneurs, ils déployèrent une formidable énergie à "sauver » les traces de pratiques qu'ils pensaient en passe de disparaître. Ceci parfois à défaut de sauver les âmes. A l'image de leur fondateur, les Croisiers partirent dans une véritable croisade pour la culture. Ils collectèrent et photographièrent des objets, organisèrent des ventes aux enchères, étudièrent le symbolisme des motifs, écrivirent des articles et construisirent des musées, dont un à Agats. Avec le temps, leur action fut de plus en plus critiquée, notamment par certains de leurs pairs dont les réactions invitent à s'interroger. On peut aussi s'enquérir du rôle de leurs amis collectionneurs dans la démarche de préservation culturelle, ainsi que sur l'intérêt trouvé par les Asmat au musée créé par d'autres qu'eux à l'intention de leurs petits-enfants, à la vente aux enchères et à l'achat massif d'objets rituellement connotés. Avant de se poser ces questions, la collecte d'objets par les Croisiers sera d'abord située dans son contexte historique et une description sera effectuée du rôle de la sculpture et du sculpteur dans la société asmat.

Cet article se base sur une combinaison de données de terrain et d'archives, collectées lors d'un séjour de deux mois en Asmat en 2001 et de trois mois en 2004 (villages simai et bismam pour la plupart, et plus accessoirement keenok et betsjm'bup), trois semaines au quartier général des pères croisiers OSC au Minnesota en 2003, une semaine chez les collectionneurs d'art Konrad à Mönchengladbach, également en 2003, et dix jours aux Pays-Bas en 2004 en compagnie des pères du Sacré-Cœur MSC.

\section{La grande rafle}

\section{Tout sauver de la jungle}

Bien que tardive, la réunion d'objets de toutes provenances dans des musées missionnaires eut lieu dans le monde entier ${ }^{2}$ mais elle se révéla souvent peu utilisable faute de renseignements ethnographiques sur les objets collectés. De grandes expositions missionnaires furent organisées à Rome, en 1925 et en 1950 notamment, pour éveiller de nouvelles vocations (O'Reilly, 1958 : 61-9). Les objets exotiques présentés à cette occasion, animés par les aventures insolites que racontaient les missionnaires euxmêmes, trouvaient inévitablement une résonance dans l'imaginaire des écoliers et des séminaristes, imprégné à cette époque de contes et de lectures choisies. La documentation lacunaire accentuait l'aura de mystère autour de ces objets et rendait d'autant plus attirant leur lieu de provenance, à la façon des cabinets de curiosité.

L'Asmat était l'une des dernières régions du monde restant à explorer. Dans les années 1950, l'établissement des Occidentaux en Asmat attira l'attention des musées étrangers qui confièrent à leurs émissaires la mission de rassembler des objets « authentiques », c'est-à-dire conçus explicitement avec des techniques et des motifs traditionnels. Autrement dit, les seuls objets dignes d'intérêt devaient avoir échappé à l'influence

2. Le père W. Schmidt, de la congrégation néerlandaise du Verbe Divin, créa un musée consacré à la NouvelleGuinée à Mödling, près de Vienne. D’autres objets se trouvent au musée de Steyl, aux Pays-Bas. Le père Schmidt fut chargé par Pie XI de diriger la section du palais de Latran consacrée aux collections missionnaires. La congrégation du Verbe Divin fonda la revue d'ethnographie Anthropos, au départ relative aux travaux missionnaires (O’Reilly, 1958 : 61-9). 
occidentale jugée préjudiciable et être façonnés à la manière caractéristique de la période anté-missionnaire ${ }^{3}$. Cependant, beaucoup d'objets étant abandonnés dans le marais après leur utilisation rituelle dans la jeuw (maison des hommes), la rapidité de leur dégradation rendait très improbable d'en découvrir de très anciens.

A partir de 1954, soit peu après l'arrivée du premier missionnaire MSC en Asmat, une partie de la sculpture fut destinée à la vente au détriment du rituel. Dans les villages d'exploitation de bois, les déchets de la coupe du bois de fer furent récupérés pour la sculpture. L'influence de l'extérieur se fit progressivement ressentir sur la production sculptée.

En 1959 accosta le premier bateau de touristes, soit un an après l'installation des premiers Croisiers. Des transactions furent conclues entre la population et les touristes, qui acquirent des sculptures par troc. D'après le Croisier Joe, les transactions se déroulaient au détriment des Asmat, qui ne recevaient qu'une pincée de tabac en échange d'un bouclier représentant des mois de travail. Par la suite, les Croisiers édictèrent une règle stipulant que les touristes devaient s'abstenir d'acheter des objets directement à la population pour plutôt s'adresser au quartier général des Croisiers, les missionnaires servant d'intermédiaires. Il en subsiste l'obligation de «premier prix » (first price) remis aux sculpteurs dont l'œuvre n'a pas été sélectionnée pour la vente aux enchères ${ }^{4}$.

Dès 1960, la renonciation prochaine des Pays-Bas à leur souveraineté provoqua le rapatriement précipité de nombreux ressortissants néerlandais, tous désireux d'emporter des souvenirs chez eux. Dans la précipitation générale, certains allèrent jusqu'à prélever des proues de pirogue sculptées (Hoogerbrugge, 1993 : 149, 152). En 1961, Michael C. Rockefeller participa à deux expéditions de rassemblement d'œuvres d'art et A. Gerbrands effectua son étude de terrain sur l'art. Certains Croisiers commencèrent à regrouper des objets asmat dans la crainte de leur disparition prochaine.

\section{La prohibition}

Déjà à cette époque, missionnaires et autorités néerlandaises tentaient de dissuader les Asmat de sculpter en raison de la connotation rituelle de cette activité. En effet, ils associaient - avec raison - les fêtes à la chasse aux têtes et espéraient, en empêchant les fêtes (donc la sculpture), dissuader les Asmat de guerroyer. En 1961, Michael C. Rockefeller disparut sur les côtes de l'asmat lors de sa seconde expédition de collecte d'objets; les circonstances de sa mort demeurèrent obscures malgré les efforts déployés

3. Cela rejoint la notion de patine citée par Appadurai (1996: 75), c'est-à-dire la propriété selon laquelle l'âge d'un bien devient un indice-clé de son haut statut et qui n'acquiert tout son sens que dans un contexte précis, en l'occurrence celui de la chasse aux têtes. La patine nourrit la capacité d'évocation des choses à la façon de la madeleine de Proust et indique qu'un mode de vie est parti à jamais, comme les Occidentaux le craignaient pour l'art asmat en raison de sa production dans un contexte de chasse aux têtes. Mais paradoxalement, il s'agissait d'une période restreinte dans le temps puisque limitée à ce que virent les premiers Occidentaux qui s'aventurèrent en Asmat.

4. L'argument justifiant le «premier prix » qui me fut avancé sur place en 2001 était que les Asmat et les acheteurs potentiels n'avaient pas de langue commune; le prix inscrit sur un papier visait donc à informer l'acheteur du prix de départ en passant outre le barrage linguistique. Une deuxième raison était de ne pas léser les Asmat, me dit-on, mais en réalité le prix était plutôt révisé à la baisse, les sculpteurs étant jugés trop gourmands par les missionnaires et par les collectionneurs. Il semble par ailleurs discutable que les intéressés n'aient pu communiquer sans l'aide du papier. 
pour l'enquête 5 . Cette mort, célèbre en Occident, fit beaucoup parler des Asmat et conforta leur réputation de guerriers sanguinaires. En 1962, les autorités indonésiennes prirent des mesures plus radicales que leurs prédécesseurs. Elles s'opposèrent à la danse, aux fêtes, au jeu du tifa (tambour ${ }^{6}$ ) et à la sculpture, supposés inextricablement liés.

Moins de dix ans après l'installation du premier missionnaire, la région asmat fut donc fermée au monde extérieur. Passages à tabac, emprisonnements pendant deux ou trois ans, autodafés des sculptures et des jeuw figurent parmi les méthodes utilisées par les fonctionnaires gouvernementaux pour contraindre les Asmat à respecter la loi nouvelle. De concert avec les autorités, les Croisiers s'opposèrent aux fêtes à cause de leurs nombreuses implications sexuelles et de la chasse aux têtes. Les lois n'allaient s'assouplir que huit ans plus tard. On peut s'imaginer qu'une si longue période suffirait aux Asmat pour renoncer définitivement aux pratiques prohibées, voire pour les oublier totalement. Sans doute aurait-il fallu que l'interdiction soit respectée, mais ce ne fut pas le cas partout ${ }^{7}$.

Entre-temps, Alphonse Sowada s'apprêtait à devenir évêque et entraînait ses collègues à suivre des cours d'anthropologie ${ }^{8}$. Dans l'Ordre croisier et ailleurs, les conséquences du concile de Vatican II (1962-5) commençaient à s'observer dans le bouleversement général des conceptions missionnaires, désormais orientées vers les populations plutôt que vers le clergé. L'assouplissement était dans l'air du temps.

En 1968, le gouvernement leva l'interdiction pesant sur plusieurs rituels dont ceux liés à l'extraction du sagou (Van Arsdale, 1978 : 318). En 1970, la confusion régnait parmi les officiels, les missionnaires et les Asmat quant au statut des fêtes (Trenkenschuh, 1982 : 36). Cette année-là, le bupati (chef de district) de Merauke commanda des mâts d'ancêtres $b i s j{ }^{9}$, sans comprendre que leur confection n'était pas dissociable d'une fête rituelle (bisj pokmbu) prohibée jusqu'alors (Trenkenschuh, 1982 : 36).

En parallèle, certains fonctionnaires gouvernementaux et négociants continuèrent à contrecarrer le déroulement des fêtes, désireux de voir les Asmat travailler dans la coupe de bois plutôt que consacrer des mois à préparer les décorations, les masques et la nourriture

5. Selon certains, il fut tué et mangé par les hommes d'Otsjanep en représailles à l'offense faite à l'un d'eux. D'autres affirment qu'il aurait perdu la vie des suites d'un naufrage lors duquel il ne put atteindre la plage à cause de la violence des vagues et des crocodiles.

6. Tambour étiré en forme de diabolo comportant une longue anse verticale sculptée et une membrane unique en peau de lézard. Jadis, la membrane était collée à l'instrument à l'aide de sang et de sperme.

7. Les interviews incitent en effet à penser que les manifestations pyromanes du gouvernement furent très localisées. Certains missionnaires assurent que les fêtes et l'activité de sculpture, loin de s'arrêter, furent simplement plus discrètes, se déroulant dans la jungle et à l'insu des missionnaires et des autorités. Trenkenschuh (1982: 35) confirme que la destruction effective des tambours et des sculptures n'eut lieu que pendant une courte période, et que les jeuw subsistèrent dans les villages en amont des rivières. Certains des Croisiers interrogés pensent au contraire que plus aucune fête n'eut lieu pendant l'interdiction et que les Asmat cessèrent de sculpter du jour au lendemain.

8. Alphonse Sowada termina ses études d'anthropologie avant d'arriver en Asmat en 1961, suivi de Dave Gallus en 1967 de Frank Trenkenschuh en 1968, qui retourna plus tard aux Etats-Unis pour achever ses études d'anthropologie en 1972.

9. Mât d'ancêtres de cinq mètres de haut en moyenne taillé dans un palétuvier retourné dont subsiste une racine qui symbolise la virilité masculine (Schneebaum, 1991 : 13-4). Van der Zee (1996 : 20) pense plutôt qu'il s'agit d'un muscadier sauvage. Ce mât commémore les décès récents de proches parents, faisant jadis office de rappel du devoir de les venger par la chasse aux têtes. 
de fête. Ces tentatives de détourner les Asmat de leurs activités de sculpture requéraient peu d'efforts dans certaines régions car à cette époque, les principaux intervenants des fêtes avaient disparu - en prison pour chasse aux têtes - et l'intérêt pour les fêtes allait en décroissant, notamment à cause de la généralisation de l'enseignement ${ }^{10}$. Si l'on voulait que les Asmat recommencent à sculpter (et tel était le souhait des nouveaux intervenants que je vais évoquer), il leur fallait un stimulant.

\section{Sculpter rend digne}

La levée de l'interdiction en 1968 coïncida avec le Programme de Développement des Nations Unies (projet $\mathrm{n}^{\circ} 18 \mathrm{FUNDWI}^{11}$ ) qui se prolongea jusqu'en 1974. Sous l'instigation des employés du projet et des missionnaires, les hommes se remirent à la sculpture à l'intention des musées et des collectionneurs occidentaux, tout en respectant les prescriptions rituelles ${ }^{12}$. Les Nations Unies achetèrent une production massive d'art asmat sous le label indigenous wood products. Dans ce cadre, les premières coopératives de bois au profit des indigènes virent le jour. En 1972, de nouveaux touristes apparurent par petits groupes.

Employé par les Nations Unies dans le cadre du projet, Hoogerbrugge (1993 : 149) décrit le rôle des Croisiers dans ce « réveil » :

\section{Les missionnaires catholiques, encouragés par Vatican II, exhortèrent les Asmat à respecter leurs traditions indigènes et s'ingénièrent à instiller un respect similaire chez les professeurs d'école actifs dans la région, qui, en général, n'étaient pas d'origine asmat (traduction $\mathrm{AdH}$ ).}

Par leurs encouragements à revenir à leurs anciennes pratiques, les Croisiers cherchaient à rendre aux Asmat leur dignité, ébranlée selon eux par l'interdiction ${ }^{13}$. De plus, ils souhaitaient obtenir pour la sculpture asmat la même reconnaissance que pour les arts orientaux, voire occidentaux. C'était une gageure pour une raison inattendue : la pudeur. Le Croisier Dave Gallus raconte qu'il espérait que le projet des Nations Unies disposerait les Indonésiens en faveur de l'art asmat, qu'ils avaient toujours considéré comme une production de seconde zone et qui offrait en spectacle à leur regard pudibond

10. Il est en effet difficile de combiner une scolarité régulière et une préparation festive de longue haleine.

11. United Nations Development Fund for the Development of West Irian.

12. L'interdiction des fêtes eut cependant diverses conséquences sur la production artistique. Sur la base des écrits du père Zegwaard, Klein (1989 : 2-3) souligne le profond enracinement de l'art dans le cycle des guerres et des fêtes, qui impliqueraient le recours à la chasse aux têtes et au cannibalisme. Cet auteur déduit de l'interdiction la désintégration de l'art et l'apparition de nouveaux reliefs plus conformes aux goûts occidentaux. Dérivés des sculptures traditionnelles, ces objets manqueraient de « vitalité brute » et de " présence charismatique », notamment à cause de l'évolution de l'outillage. A Ayam, il aurait résulté de la prohibition une perte remarquable sur le plan technique. Hoogerbrugge (1993:150-3) considère enfin que la diminution du pouvoir prêté à la sculpture - sans doute lié à la commercialisation - entraîna une perte d'intérêt pour la sculpture rituelle.

13. D'après le Croisier Jim Remmerswaal, les Asmat avaient fini par avoir honte de leurs fêtes, tournées en dérision par les étrangers et les autres groupes "civilisés », entre autres les Muyu voisins qui trouvaient ridicules leurs parures et leurs fêtes. La nudité et les coquillages passés en travers du nez étaient désignés au mépris des catéchistes, qui assimilaient les Asmat aux « hommes de la forêt » (orang hutan), dénomination très péjorative en Indonésie. Enfin, ils étaient traités d'idiots faute de s'habiller et de renoncer à la chasse aux têtes. Vu la terreur que ces chasseurs de têtes redoutés inspiraient quelques années plus tôt, on imagine l'ampleur de l'humiliation. 
la nudité des sculptures. Dans le cadre du projet FUNDWI, deux Javanais du Bureau des Affaires Culturelles furent envoyés en Asmat. Ils demandèrent « pourquoi les Asmat ne peuvent sculpter de belles choses comme les Balinais » en stigmatisant les nus, alors que l'art balinais en comportait aussi. Dès ce moment, Dave en avait déduit que la collaboration indonésienne dans le projet ne pouvait mener qu'à l'extinction de l'art asmat.

Dave relate par ailleurs comment les acheteurs commencèrent à influencer la production artistique, notamment dans le port de vêtements. Les missionnaires, les teachers et les officiels du gouvernement poussèrent les Asmat à habiller leurs personnages sculptés, comme ils durent s'habiller eux-mêmes ${ }^{14}$. Le temps passant, le nu continua à faire l'objet de polémiques. Dans les années 1980, les officiels indonésiens donnèrent leur approbation à l'organisation de la vente aux enchères à condition de dissimuler les organes sexuels, considérés comme de la pornographie. L'évêque Alphonse Sowada se serait interposé : les organes sexuels font partie de la manière dont les Asmat se représentent les ancêtres. Sa défense des représentations autochtones a des limites : l'évêque reconnaît que «c'est parfois exagéré et ça devient alors de la pornographie ».

Les Asmat purent donc continuer à sculpter leurs nus dans les limites de la décence chrétienne... à quelques exceptions près. Le frère Joe relate qu'un an après l'arrivée des missionnaires à Sjuru ${ }^{15}$, des crucifix apparaissaient avec un Christ nu. Les Croisiers expliquèrent aux gens que le Christ n'était pas nu ${ }^{16}$. " Après, ils devaient comprendre », dit Joe. Notons que la gêne à l'égard du nu n'appartient pas qu'à l'Amérique puritaine des années 1950. Dans une interview, la collectionneuse d'art asmat Ursula Konrad, amie de l'évêque avec qui elle collabora pour faire aboutir ses projets de promotion artistique, expose les plaintes récentes des touristes par rapport à la nudité des sculptures, en particulier dans l'église d'Agats : ces touristes s'offusquent de l'indécence des statues « qui appartiennent à l'Eglise catholique».

\section{Un musée dans le marais}

Du point de vue des initiatives missionnaires en faveur de la culture matérielle, c'est pendant l'interdiction des fêtes de 1962 à 1968 que les Croisiers commencèrent à rassembler des objets. Alphonse Sowada et Frank Trenkenschuh furent les principaux instigateurs d'une collecte massive d'objets dont une partie devint une des trois collections majeures d'art asmat aux Etats-Unis ${ }^{17}$. Au nom de la préservation de la culture principalement envisagée comme expression artistique (de Hontheim, 2005), les missionnaires, au cours de leurs tournées dans " l'intérieur », demandèrent à la population s'il y avait un art de bonne qualité (good art). Ils se portèrent ainsi acquéreurs de tout objet qu'ils jugèrent répondre à leur attente. Une fois étiquetés, les objets furent conservés dans un entrepôt

14. Les avis sont partagés à ce sujet. D'un côté, les Croisiers interrogés insistent sur la bonne acceptation des vêtements dès leur arrivée en Asmat, au contraire de ce qu'affirment Zegwaard et Boelaars (1982 : 28) qui soulignent la préférence des Asmat pour la nudité. Plusieurs de mes informateurs me firent part par ailleurs de l'importance de la nudité, entre autres dans certaines opérations de guérison et de mort à distance.

15. Village voisin d'Agats.

16. C'est le cas dans l'iconographie mais dans la réalité, c'est loin d'être prouvé.

17. Les collections du Peabody Museum proviennent de la collection privée du prix Nobel de médecine Dr. D. Carleton Gadjusek, qui devint collectionneur dès sa première patrouille médicale en 1959. La plupart des objets de sa collection d'art océanien, une des plus vastes des Etats-Unis, sont bien documentés (Schneebaum, 1990 : v-vii). 
à Erma ou envoyés à Agats, où l'évêque décidait de les inclure ou non dans la collection croisier. Dans ses premières collections personnelles, le couple Konrad préleva une assez grande quantité d'objets pour le projet de musée asmat; une seconde partie fut attribuée au futur musée de Jayapura. Avec l'aide des Konrad, Alphonse Sowada mit le musée d'Agats sur pied en 1973. Les objets des Konrad et une partie de la collection croisier furent réunis pour constituer l'Asmat Museum of Culture and Progress ${ }^{18}$, créé sous le patronage de la Fondation Rockefeller III et de la Fondation Asia (Konrad \& Winkelmann, 1996 : 307). Le diocèse d'Agats, propriétaire du musée, joua un rôle notable dans la continuité de l'activité sculpturale en passant commande d'objets qui manquaient à la collection du musée.

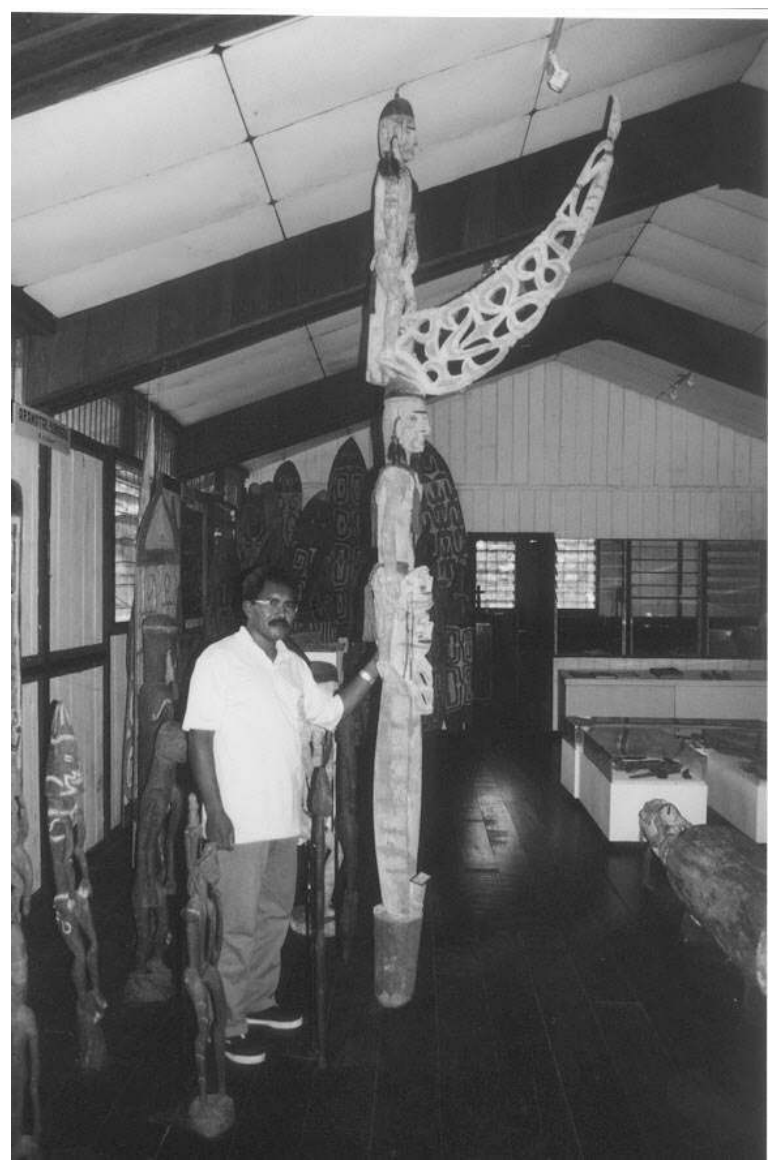

Erik Sarkol, le conservateur du musée d'Agats (C photo AdH 2004).

18. Museum Kedudayaan dan Kemajuan en indonésien. 
Le nouveau musée d'Agats nourrissait plusieurs objectifs. Becker (2000:120) écrit à propos du souhait du Croisier Trenkenschuh que les objets préservés dans ce musée permettent aux jeunes Asmat d'apprendre les procédés de fabrication de leurs aînés. Klein (1989: 3) décrit le musée comme « un centre d'éducation, où les gens en apprennent sur leur histoire et sur le monde extérieur ». Konrad et Winkelmann (1996 : 307) assurent que le musée, construit par les Asmat, est considéré par eux comme un « centre culturel». Les interviews confirment que le musée aurait eu pour but premier de faire découvrir « l'ancien style » aux écoliers. Dans l'ensemble, les Asmat interrogés sont satisfaits de savoir leur famille ou leur village représenté dans le musée, qu'ils décrivent comme un legs à leurs futurs descendants. Le frère Joe, un autre Croisier, dit quant à lui que la création du musée visait à éviter la perte du savoir-faire pendant la période de changement contemporaine de sa création; Joe pense que les Asmat en comprenaient l'utilité didactique. Lors de sa rénovation en 1993, la surface d'exposition du musée fut multipliée par trois avec l'aide de P. T. Freeport (Konrad \& Winkelmann, 1996 : 307).

\section{Enchères et sélection}

Ce musée d'Agats est le premier jalon d'une série d'initiatives promotionnelles qui allaient faire parler de l'art asmat jusqu'en Europe et aux Etats-Unis. Neuf ans après la création du musée, l'année 1981 marqua le commencement d'une autre institution en matière d'art. Sous le couvert du musée, les Croisiers ${ }^{19}$ lancèrent cette année-là un concours de sculpture avec des prix octroyés chaque année en vue de stimuler la fabrication d'art asmat (Schneebaum, 1989 : 56). Le concours fut l'occasion de lancer une vente aux enchères organisée tous les deux ou trois ans, née d'une initiative du croisier Trenkenschuh visant à récompenser les meilleurs sculptures des écoliers et de leurs pères en les proposant au musée.

La vente aux enchères proprement dite, ouverte aux acheteurs occidentaux, est précédée de deux jours pendant lesquels un jury procède à la sélection des œuvres mises en vente. Divers critères sont imposés sous peine de se voir écarter de la sélection, comme l'obligation de respecter les «techniques traditionnelles ${ }^{20}$ » ou de s'abstenir de détails « modernes » ou « religieux » (même si l'évêque reconnaît et entérine le changement, tant dans la société que dans l'art). Cependant, ces sculptures abondent dans la réalité. Dans la sculpture, des éléments asmat se mêlent à la croix et à des scènes de la Genèse, exprimant la compréhension asmat du royaume de Dieu. L'évêque déclare que l'art religieux chrétien fut écarté de la vente aux enchères ${ }^{21}$ supposée afficher « la vision asmat

19. Le film de Schneebaum (2000) désigne la vente d'Agats comme une Annual Intertribal Auction. Muet sur le rôle des missionnaires, Schneebaum déclare : «we had this auction in Agats » sans préciser qui est le «we ». Alphonse Sowada conteste tout rapport entre Schneebaum et l'organisation de la vente.

20. En 2001, les responsables de la sélection se montrèrent particulièrement attentifs au soin apporté au travail et aux pigments utilisés, comme pour les tifa (tambours) que certains étaient tentés de foncer avec de l'huile de vidange de «Johnson» au lieu d'être noircis au feu ou de les enterrer pendant quinze jours dans de la « bonne boue ». Un habitant d'Ewer me signala que ce dernier traitement ne pouvait être appliqué qu'au « bois de fer». Selon Geremias, un des organisateurs, le bois blanc de mangrove (kayu putih) ne s'utilise que pour les sculptures « ayant un sens » tandis que le bois de fer, moins fragile, est réservé aux objets à vendre aux Indonésiens.

21. Une scène sculptée représentant Adam, Eve, Dieu et le serpent se trouve au musée d'Agats, mais c'est la seule sur laquelle on trouve des éléments chrétiens. 
du monde » (le « royaume de Dieu », apparemment, n'en fait pas partie). De son côté, Ursula Konrad ne s'étonne pas de l'absence d'éléments chrétiens dans la sculpture asmat : «ce n'était pas le but ». Les quelques objets inspirés de christianisme qu'elle possède furent acquis via la mission, et non pas dans les villages. Dans les villages en effet, rares sont ceux qui sculptent des objets ayant un quelconque rapport avec le christianisme : je n'en rencontrai qu'un à Uwus qui me montra des crucifix de sa fabrication. Sinon pas de croix, pas de Christ, pas de Marie, pas de scène de la Bible hormis dans les églises. De nombreux sculpteurs se déclarèrent capables de produire ce type de réalisation, mais sur commande.

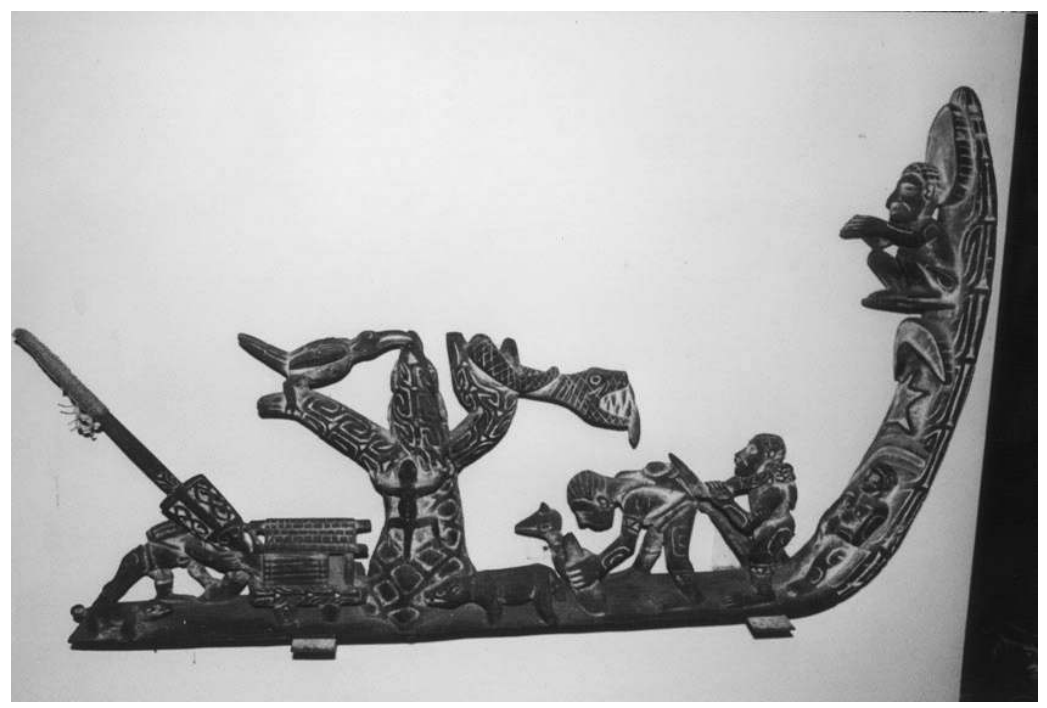

Scène sculptée de la Genèse exposée dans le musée d'Agats : au-dessus du firmament, Dieu regarde Eve, Adam et le serpent (C) photo AdH 2004).

Les œuvres de la vente, qui sont présentées en Occident via le catalogue assemblé après coup par les Croisiers et les Konrad, sont supposées représentatives de la culture asmat, figée dans la vision qu'en ont les organisateurs de la vente. Désireux de voir élire une de leurs sculptures, tant pour des questions pécuniaires qu'honorifiques, les Asmat sont amenés à se rallier à ce point de vue que leurs représentants défendent également. Ils ont la conviction non fondée qu'en plaidant ainsi pour l'immuable, ils défendent la continuité de la culture et la préservation du peuple asmat. Pourtant, en dépit des constantes de forme propres aux villages ou aux « groupes culturels » (Konrad \& Winkelmann, 1996 : 316) qui déterminent des modèles classés par aires géographiques, l'évolution des techniques et des sujets est inévitable. Beaucoup d'œuvres s'agrémentent de géométries inventives et nouvelles, à en juger par l'étonnement des membres du jury lors de la sélection. J'entendis des remarques de type : « telle caractéristique est traditionnelle et non telle autre », «j'ai vu ceci il y a vingt ans à un tel endroit », etc. Par conséquent, les qualités esthétiques ne suffisent pas. Fondant pour une assiette à chaux ornée de motifs inédits, Ursula Konrad insista trois fois pour la sélectionner en 2001 mais les deux étudiants asmat du jury n'en démordirent pas : ce n'était pas traditionnel. 
Quelle que soit la méthode pour les en convaincre, les craintes que les Asmat renoncent à leur artisanat n'ont plus lieu d'être. Avec l'instauration de la vente aux enchères et l'appui des missionnaires qui enrichissent fréquemment leur collection personnelle, l'activité artistique a acquis une place prépondérante dans les villages. Le père Virgil confirme que l'art connaît une vigueur nouvelle, encouragée par les relations mercantiles. Lui-même le favorise à petite échelle : je le vis acheter une sculpture au prix annoncé à un artiste qui se présenta à sa porte. Le nouvel évêque du diocèse Agats-Asmat, Alo Murwito OFM, partage son enthousiasme, et dans sa maison s'accumulent de nombreux objets.

\section{L'art asmat montré aux Américains}

Les efforts des Croisiers et de l'évêque Alphonse pour stimuler l'art asmat ne se limitèrent pas au musée et à la vente d'Agats. Quand Alphonse devint évêque en 1969, le provincial lui demanda d'envoyer des objets asmat aux Etats-Unis, ce qu'il fit. Dans un premier temps, il envoya ses sculptures à la mission des Croisiers à Hastings dans le Nebraska, puis y fonda le Crosier Asmat Museum dont les collections furent exposées à plusieurs reprises. L'American Museum of Asmat Art de Shoreview fut créé en 1994 avec Tom Carkhuff comme directeur. Mary Braun en fut nommée directrice cinq ans plus tard. Le conseil d'administration du musée est actuellement indépendant, bien que composé de plusieurs membres croisiers.

L'attention portée à la promotion de l'art asmat donna lieu à d'autres initiatives. En 1984, une exposition de sculptures asmat donna lieu à une démonstration de techniques par quinze sculpteurs à Jakarta. Sous la direction de l'épouse du président Suharto, une Fondation Asmat fut créée deux ans plus tard. Puis une tournée de danse asmat eu lieu en Europe en 1987 (Hoogerbrugge, 1993 : 150). De même titre qu'un ouvrage de Schneebaum (1990), la grande exposition Embodied Spirits fut montée en 1990, en collaboration avec le Peabody Museum de Salem et le Crosier Asmat Museum. En 1991, 29 hommes (dont Yuven Biakai) et une femme asmat partirent pour une tournée américaine qui fut produite dans quatre villes, dont New York au Metropolitan Museum of Art.

Dans la continuité de la vente d'Agats, les Croisiers mirent sur pied plusieurs ventes aux enchères au profit des musées de Shoreview et d'Agats. Une Benefit Auction (vente de charité) for Bishop Sowada Gallery of Asmat Art eut lieu en juillet 1998 avec la présence de Sotheby's. La vente suivante eut lieu au National Art Club de New York en mai 1999. En plus des ventes aux enchères, les pères croisiers installés en territoire asmat organisèrent des tours touristiques, notamment pour les membres de la Sago Worm Society $^{22}$. Le film de Schneebaum (2000) montre également une Annual Asmat Benefit Auction au Metropolitan Museum of Art à New York, où un bouclier se vend 5900 euros, soit à un prix nettement supérieur à ceux que l'on pratique habituellement à la vente aux enchères d'Agats.

22. Fondée par Dave Gallus et le directeur de mission de son diocèse, cette société comporte environ 300 membres de la jet set américaine, dont le chanteur Mick Jagger, Colleen Needles, la réalisatrice d'un film sur les Asmat (1994), et Jim Olsen, un des Fund Raisers des Croisiers. Y sont admis ceux qui ont consommé des larves de capricorne, dont les Asmat sont friands. 


\section{Les collections appartiennent aux Asmat}

Tom, l'ancien directeur du musée de Shoreview, déclare qu'une partie de la collection du musée est officiellement possédée par les Croisiers américains et par le diocèse d'Agats. Quand il était directeur, une convention légale fut passée entre le diocèse et les Croisiers pour céder la collection en leasing au musée pendant 99 ans. Cette convention comporte quelques restrictions sur l'usage qui est fait des œuvres. Fondus dans la collection du musée, des objets d'une autre provenance, le Sepik, étonnent par leur présence insolite sur cette surface d'exposition consacrée à l'art asmat. Il s'agirait de legs. Dans le catalogue des ventes aux enchères organisées par les Croisiers, des objets du Sepik sont proposés aux côtés des objets asmat, comme dans l'ouvrage comparatif du Minnesota Museum of Art (1989). Mary, la directrice du musée, m'affirma que tout ce qui était montré appartenait aux Asmat, comme s'il s'agissait d'un prêt, et qu'ils pourraient tout récupérer quand bon leur semblerait.

Préoccupé par l'organisation du musée de Shoreview, Dave voudrait impliquer davantage les Asmat et les y inviter de temps à autre. Interrogé dans un bulletin de l'American Museum of Asmat $\mathrm{Art}^{23}$, Dave insiste sur l'importance de maintenir le lien entre un objet et son contexte culturel :

Si l'art asmat est soustrait à sa relation d'avec les Asmat, je pense que l'on vole les Asmat de leur valeur la plus précieuse, leur rapport aux ancêtres et leur rapport à l'identité. Je pense que la rupture entre la culture asmat et leur art contribue à un génocide culturel (traduction $\mathrm{AdH}$ ).

Les activités du musée suscitent des avis divergents. Les uns vantent les qualités d'une société asmat en mutation permanente et les autres le maintien (inespéré) de traditions datant du néolithique. En dépit de sa création par des prêtres instruits en anthropologie, le musée transmet à ses membres des textes dont la teneur est parfois édifiante. Jointe au dernier numéro du bulletin, une publicité pour un tour chez les Asmat et les Dani « offre au voyageur aventurier l'opportunité de faire l'expérience de tribus émergeant de l'Age de la Pierre et se cramponnant encore à leurs anciens rituels ».

\section{Le sculpteur dans son contexte}

\section{Le premier sculpteur}

Il est temps d'évoquer le rôle de la sculpture dans la société asmat, et en particulier dans l'histoire orale. D'après un mythe raconté dans nombre d'ouvrages sur les Asmat, les premiers objets furent sculptés par Fumewripitsj, dit «l'homme du vent » créateur, sauvé de la noyade par un aigle. Il construisit une jeuw et, se sentant solitaire, abattit des arbres pour sculpter des silhouettes humaines. Après les avoir installées dans sa maison de fête, il les anima en jouant du tambour qu'il sculpta ensuite. Les œuvres se mirent à chanter et à danser : ce furent les premiers Asmat. Puis Fumewripitsj tua un crocodile et le coupa en morceaux. Les fragments, jetés vers le ciel, retombèrent sur la terre et se transformèrent en d'autres êtres humains : les voisins des Asmat. Plusieurs auteurs déduisent de ce mythe que le bois est la matière originelle, les Asmat se nommant eux-

23. Vol. $7, \mathrm{n}^{\circ} 3$, mars 2003, p. 3 . 
mêmes « hommes de l'arbre » (as asmar) ou « hommes vrais » (asma ow) ${ }^{24}$. D'après Helfrich (1996: 43), « chaque acte artistique est tout autant un acte religieux »; l'acte de sculpture commémorerait la création du premier ancêtre.

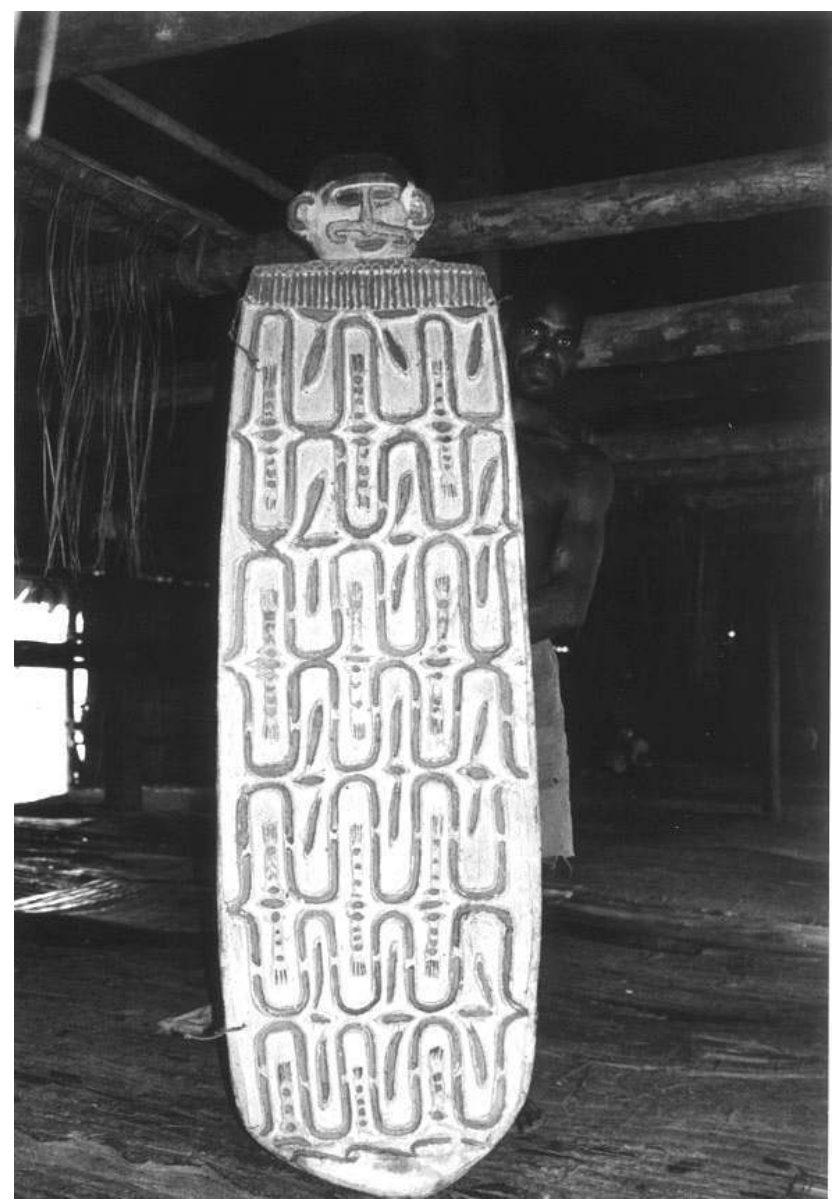

Comme celles des autres sculptures de la maison des hommes d'Amborep, l'histoire de ce bouclier est incluse dans les mythes fondateurs de la société asmat (C) photo AdH 2004).

24. Il y est fait allusion dans le titre de l'ouvrage de Schneebaum (1989) : People of the River, People of the Tree : Change and Continuity in Sepik and Asmat, les " gens de la rivière » étant ceux du fleuve Sepik et ceux de l'arbre, les Asmat (cf. aussi van der Zee, 1996 : 76). Sur le terrain cependant, mes informateurs asmat contestent unanimement l'origine forestière de leurs ancêtres; la matière originelle dans laquelle les Asmat ont été façonnés, la boue, était jadis aussi le lieu où l'on coulait tout ou partie du cadavre respectivement lors de premières ou de secondes funérailles dans les régions côtières. Il convient de se demander si ce retour à la terre mère n'est pas né de l'influence du christianisme, en allusion à la promesse divine faite à Adam dans la Genèse de se retransformer en poussière après la mort. 
A l'examen des données de terrain, un bémol s'impose. D'après mes informateurs asmat, Fumewripitsj, loin d'être le créateur des Asmat, fut plutôt celui des Mimika, leurs voisins. "Les missionnaires et le conservateur du musée n'y connaissent rien ", m'ont assuré plusieurs conteurs. Dans les mythes collectés principalement à Amborep et à Sjuru, Fumewripitsj apparaît être un personnage secondaire : Beworpitsj était le premier. Beworpitsj était aussi le premier sculpteur, et une lance de sa fabrication figure encore dans les trésors de la jeuw d'Amborep. A l'analyse, Fumewripitsj se révèle un héros d'épopée dans l'ombre de Beworpitsj, dont la conduite justifie nombre d'institutions clés de la société asmat - telles que la guerre et la chasse aux têtes - dans les nombreux mythes dont il est le héros. Beworpitsj est enfin présenté aux enfants comme un modèle de comportement, au contraire de Fumewripitsj.

\section{Un pilier de la maison des hommes}

Les divergences de points de vue sur le plan des contenus mythiques n'ôtent en rien le caractère essentiel de l'acte de sculpter. le sculpteur wow'ipitsj fait partie des membres du wair, le centre physique de la jeuw, délimité par des nattes en feuilles de pandanus sur lesquelles les membres s'asseyent autour de l'âtre (yowse) principal. Dans le wair, les ancêtres sont présents en permanence; à Ayam, certains wair 'ipitsj se déclarent habités par eux dès qu'ils pénètrent dans la jeuw et se sentent dès lors " plus lourds ». Le « grenier », une plate-forme formant un second plancher à hauteur d'homme et servant au rangement, abrite divers objets composant le patrimoine ancestral et dont les mythes du village retracent l'histoire. Les wair 'ipitsj comprennent des sculpteurs, des chanteurs, des joueurs de tifa (tambour), des tasma'ipitsj (guerrier attesté habilité à déclarer une interruption de fête) et des conteurs, ces fonctions pouvant être cumulables. Ils sont les détenteurs officiels de la tradition et consomment les offrandes faites aux ancêtres par les autres hommes de la jeuw. Par leur intermédiaire, l'offrande prend corps dans les descendants de ceux à qui elle est destinée. Comme ses homologues wair'ipitsj, le wow'ipitsj est en contact avec les ancêtres de qui il tire sa science; ce sont eux qui lui transmettent l'image mentale de ce qu'il va sculpter. Avant même de se mettre à l'ouvrage, il a en tête un ou plusieurs projets et sait d'avance à quoi ressemblera le produit fini.

Enfin, le réalisateur de film Corillion (2001) prête aux sculpteurs une fonction politique. Outre leur position stratégique dans la jeuw, qui leur permet de s'exprimer et de prendre des décisions au nom des ancêtres, les wair'ipitsj en général jouent un rôle crucial dans la gestion du temps villageois, organisé à partir de la jeuw. Une fête doit être interrompue en cas de maladie de l'un d'eux, et on les remplace immédiatement en cas de décès. Selon Markus Yisimamtsji de Atsj, certains sculpteurs détiennent un don supplémentaire qui les fait craindre : ils sont capables de concevoir la magie de mort, l'arow'pok, dans une version particulière qui s'intègre à la sculpture et qui a généralement pour but de se débarrasser d'un enfant ou d'une épouse encombrante.

\section{La relève}

Sur le plan de la formation, les apprentis bénéficient de l'enseignement d'un sculpteur réputé de leur village, pas nécessairement de la même famille bien que l'on trouve des dynasties de sculpteurs. Inévitablement, les formes évoluent à travers le temps, malgré l'encouragement des organisateurs de la vente aux enchères à maintenir les sculptures dans un moule jugé « traditionnel ». La compétence s'acquiert par l'expérience et non 
par l'appartenance à une caste, mais l'empreinte de la région ou du village d'origine est d'ordinaire reconnaissable par les spécialistes ${ }^{25}$. D'après Schneebaum (1991 : 81-2), seuls les meilleurs sculpteurs sont sollicités pour réaliser des objets rituels tels que tambours, boucliers ou figures d'ancêtres. La dextérité peut être précoce; en 2001, les lauréats à la vente aux enchères d'Agats comptaient un garçon de quatorze ans.

Yufen Biakai, le kepala suku ${ }^{26}$ d'Agats et ancien protégé de l'évêché, assure que «n'importe qui peut devenir sculpteur» mais déplore la baisse de l'habileté des sculpteurs d'année en année, à proportion du temps croissant consacré à la pêche ou au gaharu ${ }^{27}$. Il semble que cette plainte se fasse l'écho du discours occidental sur la sculpture, même si certains Asmat partagent les mêmes doléances ${ }^{28}$. Dans le même ordre d'idées, la ressemblance frappante entre les textes de Yufen et les écrits missionnaires, ces derniers étant principalement axés sur les traditions du groupe keenok de Sawa-Erma, invite à soupçonner une influence de l'un sur l'autre. Interrogé sur la fiabilité de ses connaissances sur " la tradition » asmat, Yufen, faute d'en faire partie, conteste le rôle essentiel des détenteurs de la tradition wair 'ipitsj, qu'il qualifie de «pauvres villageois ignares » par rapport à lui, qui a reçu une instruction ${ }^{29}$.

\section{Au cour de l'objet}

Sur le plan technique, les objets sculptés ou tressés sont souvent trichromes : le pigment rouge provient d'une variété de terre riche en hématite (Helfrich, 1996:38), le blanc est obtenu à partir de coquilles d'escargots de rivière concassées (ou de corail dans les villages côtiers) et le noir avec de la suie et du charbon de bois. Les pirogues sont peintes à l'intérieur et à l'extérieur. Nombre d'objets sont peints, y compris la vannerie (sacs portés dans le dos, costumes des masques, coiffes, panneaux décoratifs destinés aux touristes) dont le tressage délimite les zones géométriques à mettre en couleur. Traditionnellement, les objets sont sculptés en bois blanc léger (pit, ou kayu putih en indonésien) ou dans des

25. Pour ne citer que lui, Schneebaum (1990:30) répartit la production artistique en quatre aires stylistiques : les côtes centrales et sud, le nord-ouest, les villages « citak» de l'est et les alentours du fleuve Brazza et les piémonts du nord-est. Les poteaux d'ancêtres bisj ne sont par exemple fabriqués que dans une dizaine de villages de l'asmat central. Dans des groupes de villages de l'ouest reliés entre eux par la parenté, on trouve des représentations de crocodiles, des récipients à larves de capricorne, des figures de guerriers tués au combat, ou des panneaux décoratifs destinés à la vente comme ceux, caractéristiques, de Sawa-Erma. Certaines populations ne façonnent pas de figures d'ancêtres, comme les Citak et les Brazza.

26. «Chef ethnique » parfois consulté par le gouvernement indonésien en tant que représentant « ethnique » d'un village, par opposition au kepala desa, qui connaît la langue indonésienne et rend compte aux autorités de sa gestion du village.

27. Aquilaria spp., bois d'aigle ou bois d'encens que l'on collecte en quantités en asmat en vue de sa revente notamment en Arabie Saoudite.

28. De temps à autre, un ancien se plaint de ce que « les jeunes ne sont plus capables d'un travail de qualité ». A Sjuru, Harun accuse les jeunes d'être « incapables de sculpter de manière traditionnelle malgré leur bonne volonté »; Virgil attribue son opinion à sa religion protestante qui nie toute valeur positive aux manifestations visuelles de la période anté-missionnaire, dont la sculpture. Schneebaum (1989 : 52) pense que le caractère sacré des outils et des œuvres échappe à la plupart des jeunes, qui ne voient plus dans la sculpture qu'une source de profit.

29. A cet égard, les opinions de Yufen et son élection comme chef de la LMAA (ou Lembaga Musyawarah Adat Asmat), une association créée en 2000 en vue de la défense des droits des Asmat, sont loin de rencontrer l'unanimité parmi les Asmat, comme l'on peut s'en douter. 
bois spécifiques pour les bisj (tow, ou kayu palah hutan en indonésien) ou les pirogues (tsjii'osj), mais le succès du « bois de fer » (Intsia spp., kayu besi en indonésien) rouge et lourd auprès des acheteurs indonésiens incite de plus en plus de sculpteurs à le préférer au bois blanc, très fragile. A cet égard, un tabou frappant l'abattage de ce bois a pour origine la présence d'un esprit féminin au cœur de l'arbre (pasj'khomerawtsj), auquel il est prescrit de faire une offrande sous peine de danger pour les hommes qui s'aventurent dans son voisinage. La résistance de ce bois à la putréfaction, qui le fait préférer aux autres essences dans la construction, justifie la pression extérieure pour adapter les comportements locaux aux impératifs économiques. Désormais, ces arbres sont abattus après offrande et excuses préalables. Comme les autres arbres, l'arbre de bois de fer (pasj'osj), une fois sculpté, change de nom (pasj'kawee) : la terminologie évolue en effet selon le degré de maturation.

Sur le plan des motifs, le bipane, l'ornement masculin formé d'un coquillage taillé en double spirale glissé dans le nez, demeure dans le motif en volute couramment sculpté sur les boucliers; un autre motif utile à la guerre, pour son aptitude à terrasser l'ennemi de frayeur, est l'ainor, deux spirales contiguës au milieu cambré (Schneebaum, 1991 : 79-80). Comme il n'y a plus de guerre, ces motifs, comme d'autres relatifs à la chasse aux têtes, conservent essentiellement leurs qualités d'ornementation et de mémoire des ancêtres. Citons aussi la spirale évoquant la queue d'un couscous et un motif floral en éventail dérivé des racines d'un banian (van der Zee, 1996 : 75).

S'intéresser à l'apparence de la sculpture n'est qu'une manière de la comprendre. Dans la plupart des cas, le sculpteur crée un objet vivant, susceptible de rancune. Ainsi, la négligence à l'égard d'une sculpture doit automatiquement donner lieu à des excuses, sous peine de subir la vengeance de l'objet bafoué. Le sculpteur confie d'ailleurs son œuvre à son acquéreur en lui recommandant d'en prendre soin, comme d'un être vivant. De la même manière, des villageois de diverses provenances m'affirmèrent que tout objet façonné par un Asmat, sculpté ou tressé, est habité par une âme ${ }^{30}$ (dambuw, ou nyawa en indonésien) dont les déambulations nocturnes sont clairement audibles. Markus Yisimamtsji confirme que la nuit est le moment de la libération des esprits des choses; dans son village d'Atsj, des sculptures sont regroupées en un lieu parfois tellement bruyant que l'on se croirait, dit-il, au milieu d'une foule.

Une fois dans l'objet, la dambuw peut cependant en être délogée. En effet, un objet incomplet « perd» l'esprit qui l'anime ${ }^{31}$. Normalement, l'art sculpté n'est pas destiné à subsister en dehors de ce pour quoi il a été conçu; en quelque sorte, le morceau prélevé empêche l'objet de poursuivre une existence ailleurs que dans le village d'origine. A Agats, Geremias M'Baith explique qu'en mémoire des ancêtres, les sculptures rituelles - c'est-à-dire celles qui font l'objet de fêtes - sont identifiées à des humains décédés et traitées comme eux jusqu'à ce que leur mort ait obtenu réparation; elles peuvent alors être

30. Ce terme désigne également l'âme humaine, qui quitte le corps pendant la maladie (le rôle du guérisseur consiste à rappeler l'âme) et qui s'évanouit dans le néant après la mort. Par contre, certains objets, animaux et végétaux au rôle-clé dans la société sont habités par un autre type d'âme, plus judicieusement traduit par « esprit », capable d'actions - plus complexes que la dambuw - dictées par les ancêtres et reconnaissable par une terminologie distincte qui ne prend son sens qu'en association avec un autre terme (... 'khomerawtsj).

31. Cela ne se vérifie pas toujours. J'ai pu voir de nombreux objets de valeur non rituelle mais décorative (clairement destinés aux touristes) et qui n'avaient pas été amputés d'un morceau. 
abandonnées ou destinées à la vente et sont justement reconnaissables parce qu'on leur a ôté un morceau. Une pratique évoquée par Konrad et Winkelmann (1996 : 304) illustre cette « insécabilité » des objets. Ces auteurs racontent qu'après la fête, les membres des mâts d'ancêtres à Uwus sont amputés et abandonnés dans la jungle pour empêcher les esprits d'y rester.

Schneebaum (1989: 51) rejoint Geremias lorsqu'il affirme que les sculptures sont l'incarnation des morts. Il faut reconnaître que malgré leur commercialisation massive, les sculptures rituelles restent nécessairement présentes à toutes les fêtes et prennent vie lorsqu'elles sont baptisées du nom d'un homme mort récemment (Schneebaum, 1990 : 26), supposé lui prêter son pouvoir. Jadis, un bouclier ainsi nommé conférait du courage au descendant du défunt et lui assurait la victoire ${ }^{32}$ (Schneebaum, 1991 : 81). Les sculptures anthropomorphes se voient d'office attribuer le nom d'un ancêtre (van der Zee, 1996 : 24), mais aussi les pirogues, les maisons, les pagaies, les lances, les sacs de fibres, des colliers de dents de chien, les chiens domestiques et les cochons. Ces objets sont généralement appelés etsjo'pok, « les choses qui font grandir ». Jadis, ils rappelaient le devoir des hommes de partir en raid pour venger leurs morts (Zegwaard, 1959 : 1029).

\section{L'art asmat n'existe pas}

$\mathrm{Au}$ vu de la fonction rituelle de la sculpture asmat, on pourrait se demander ce qu'il en est du rapport entre la sculpture et « l'art» tel qu'il est compris en Occident. L'étude du vocabulaire est assez révélatrice sur ce plan, tout comme l'est celle des interprétations des auteurs qui se sont penchés sur le sujet. D'après Schneebaum (1989 : 52), les Asmat ne considèrent pas leurs sculptures comme de l'art ni les sculpteurs comme des artistes : j'aurais tendance à me ranger à cet avis. En parallèle, la terminologie est loin de rencontrer l'approbation générale. Selon Schneebaum (op. cit.), le sculpteur est un tsjestsju'ipitsj (qu'il traduit par " homme intelligent») ou un wow'ipistj (homme dessinateur). Sans leur donner la même signification, Helfrich (1996 : 43) distingue également le wow'ipitsj (créateur) du tsjesju'ipitsj (homme inspiré) et souligne le statut privilégié des sculpteurs. Dans une interview, Ursula Konrad effectue une distinction comparable. Lorsqu'elle se fait énumérer les sculpteurs (penukia) en arrivant dans un village, elle obtient entre vingt et trente noms. Parmi eux, seuls un ou deux sont tsjesju'ipitsj, sculpteurs traditionnels. D'après Alphonse Sowada, le terme tsjestsju'ipitsj s'appliquerait plutôt aux chefs et se traduirait par « homme dont les aptitudes supérieures sont reconnues ».

Sur le terrain, les entretiens avec les Asmat invitent à prendre du recul par rapport à ces postulats. Le sculpteur est dit wow'ipistj, «l'homme bouclier », le bouclier étant l'objet sculpté par excellence. Le bouclier ne peut être confectionné que dans ces circonstances précises, dans la même essence (tsjii'osj) que celle de la pirogue, et son existence est obligatoirement consacrée par une fête. En Asmat, le sculpteur est donc wow'ipitsj tandis que le tsjestsju 'ipitsj, moins souvent cité, apparaît plutôt dans le discours des informateurs

32. A cet égard, les entretiens sur le terrain indiquent l'obtention systématique de la victoire par le village qui part en raid, tout comme le village attaqué vaincra lorsqu'il partira en représailles. Préalablement, les organisateurs du raid se protègent par un sort de victoire (eeram'pok) accompli à un endroit sacré avant de s'aventurer jusqu'au village ennemi. L'eeram est un sort aux facettes multiples dont la connaissance peut se recevoir comme cadeau d'initiation; actuellement, ses vertus thérapeutiques semblent les plus sollicitées. Il est mis en œuvre par un des deux principaux types de guérisseurs, l'eeram 'ipitsj, par opposition au damer 'ipitsj, qui joue aussi un rôle d'exorciste. 
comme un terme générique désignant un homme possédant quelque talent. La notion d'art invite également à s'interroger. Consultée sur la question, Ursula Konrad ne connaît pas de traduction pour la notion « art »; selon elle, il n'existe pas de terme désignant un « mot général », comme celui de travail, par exemple. Les mots utilisés par les Asmat actuellement sont empruntés de l'indonésien. Alphonse Sowada déclare quant à lui qu'il existe bien un mot asmat signifiant « art ». Cependant, sa formation en anthropologie l'a probablement sensibilisé à l'absence de vocabulaire sur l'art dans de nombreuses sociétés non occidentales, et pourrait lui avoir fait adapter sa traduction. L'expérience de terrain a de toute façon tendance à montrer que les seuls à parler « d'artiste » sont extérieurs à la société asmat.

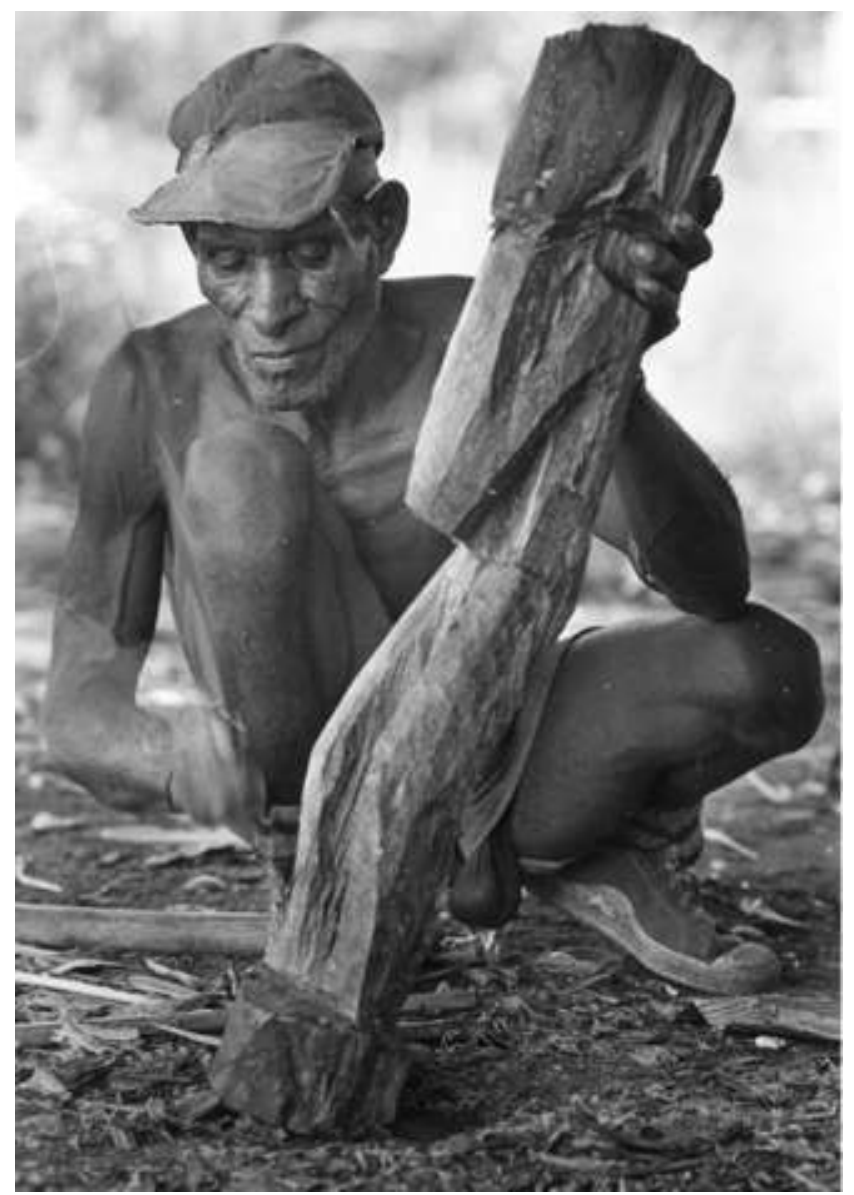

Sculpteur au travail (Photo d'archives des pères croisiers, Shoreview, 2003).

L'absence de connotation artistique dans la définition du sculpteur est loin d'atténuer son importance, comme nous l'avons vu. Pour Konrad et Winkelmann (1996 : 36), l'habileté de « l'artiste » (qu'ils traduisent par le terme vague de tsjesju'ipitsj) est reconnue par la société sur qui il exerce une puissante influence. Il peut assumer quatre rôles : chanteur, joueur de tambour, narrateur de mythes et sculpteur. Et surtout, il aurait le pouvoir de 
donner une âme aux choses. Il semble que ces auteurs confondent le tsjesju'ipitsj et le wair 'ipitsj, ce dernier étant effectivement susceptible d'assumer les rôles précités et dont l'influence sur la société est incontestable. Dans la littérature occidentale sur les Asmat citée précédemment, il lui manque pourtant une dimension fondamentale : celle d'un maillon entre le monde des vivants et celui des ancêtres, que le wair'ipitsj prouve en siégeant dans le wair et en consommant les offrandes faites aux ancêtres collectivement et en leur nom.

\section{Quand le poteau d'ancêtres devient un objet de lucre}

\section{Selon l'inspiration des collectionneurs}

A plusieurs reprises, j'ai cité le couple Konrad et plus particulièrement Ursula Konrad, une amie de l'évêque Alphonse Sowada - présentée sur le terrain comme « sa cousine »- avec qui elle collecta des objets asmat au nom d'un souci commun de préservation de la culture. Son intervention dans la production artistique ne date pas d'hier : dans un entretien, elle se targue de s'être consacrée depuis 33 ans aux Asmat et à la diffusion de l'art. En effet, le projet des Nations Unies suscita l'arrivée d'étrangers qui s'étaient vu refuser l'entrée dans la province indonésienne dont un couple d'Allemands, Gunter et Ursula Konrad. Lors d'une expédition zoologique en 1971 pour observer les oiseaux de paradis, l'un et l'autre se découvrirent une passion pour l'art asmat. Cela prit des proportions gigantesques. D'année en année, ils accumulèrent ce qui constitue actuellement la collection Konrad au Musée d'Ethnologie à Heidelberg et la galerie Konrad à Mönchengladbach.

Depuis 1971, donc, les Konrad consacrent une grande partie de leur temps libre aux Asmat. Outre le rassemblement d'objets, ils suscitèrent l'intérêt de cet art à travers leurs publications. En Allemagne, la promotion se concrétise de manière inattendue. Dans la galerie Konrad, aux côtés de scènes de la vie quotidienne dans le genre musée d'ethnographie à l'ancienne, Ursula expose les œuvres d'artistes allemands confectionnées à partir d'objets asmat réels ou imaginaires. Parmi elles, des cubes de paraffine jaune imprimés de motifs de vannerie asmat côtoient des «poteaux d'ancêtres » peints de couleurs vives et sur lesquels figurent une blonde en jeans, un ordinateur et une machine à laver. Des statues asmat sont récupérées pour construire de nouvelles œuvres, à côté de photographies prises «à l'instant» sur le terrain. Des panneaux asmat tressés sont découpés en lanières et figés dans la paraffine pour former des treillis mis sous cadre. On trouve aussi des peintures très colorées de visages asmat déformés inspirés de photos en noir et blanc, ou encore des groupes de petites bûches verticales surmontées par une tête sculptée comme cela se fait dans la région d'Agats. Un des artistes est réputé « travailler avec les esprits ».

Dans l'arrière-boutique, d'énormes entrepôts débordent d'objets, des pirogues des âmes entassées sur de hautes étagères aux masques des esprits suspendus au plafond à des crochets comme dans une garde-robe. Les Konrad ont vu grand : quand ils venaient en Asmat, ils remplissaient les conteneurs de la mine de P. T. Freeport ${ }^{33}$ à destination

33. Cette compagnie minière américaine exploite la plus grande mine de cuivre du monde à Tembagapura depuis 1973. En échange du service religieux, les Croisiers bénéficient de l'aide financière et du réseau de transport de Freeport; c'est vraisemblablement grâce à leur aide que les Konrad ont pu en profiter aussi. 
de l'Allemagne. Alphonse Sowada surnomme d'ailleurs Ursula la «mother of the company ». Elle sait sélectionner ses relations : une banque compte parmi ses sponsors, et les artistes promus par l'intermédiaire de la galerie et des expositions sont ses amis. La promotion d'art asmat fonctionne ainsi à la manière d'un réseau, avec ventes publiques et vernissages d'expositions auxquels est invité un public choisi et où se mélangent l'art contemporain allemand et « l'art asmat ». Ce mélange se retrouve tant à l'intérieur de la maison des Konrad que dans leur musée. Des poteaux asmat décorent les parcs publics de Mönchengladbach et l'extérieur de l'hôpital que dirige Gunter ${ }^{34}$. D'autres poteaux pourrissent dans leur jardin, comme si on ne savait plus quoi en faire.

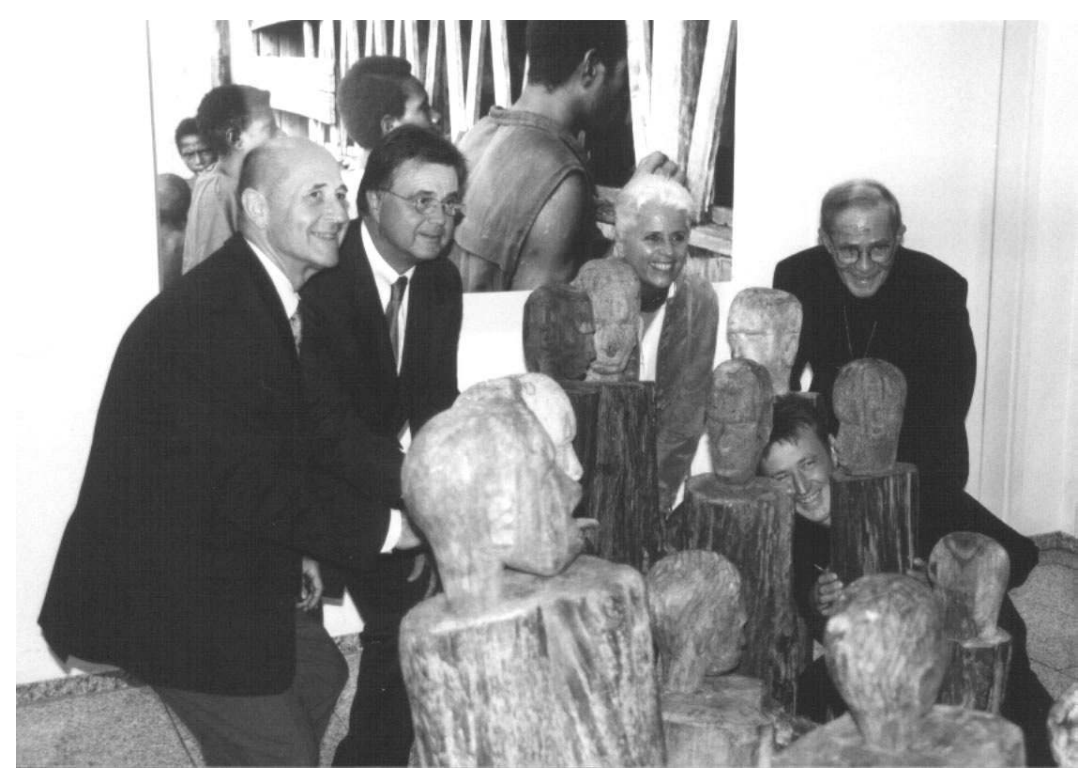

Le couple Konrad (Gunter Konrad à gauche) et Alphonse Sowada (à droite) lors d'un vernissage d'une exposition de photos à Mönchengladbach (C) photo AdH 2003).

Selon les déclarations d'Ursula, le but de cette opération d'envergure est d'aider les Asmat. Dans la mesure du possible, elle essaie d'intégrer les sculpteurs à son action. Outre la publicité, cette action consiste principalement à passer commande et à « travailler avec les artistes » c'est-à-dire à observer leurs réactions devant des œuvres contemporaines allemandes inspirées ou non par l'art asmat. Lors de mon interview de 2003, Ursula voulait mettre en présence un artiste allemand et un artiste asmat pour leur permettre de confronter leurs idées; une autre intention était de montrer des coiffures de tresses africaines aux femmes. Quand on lui fait observer que ces procédés rendent la part d'intervention de l'artiste difficile à déterminer, Ursula exprime qu'elle n'a pas eu

34. De métier, Gunter est un urologue réputé spécialiste de la microchirurgie et Ursula a une formation de modiste, ce qui aurait contribué d'après Alphonse à affiner sa sensibilité en matière d'art. 
d'influence sur la sculpture. Elle incrimine plutôt les missionnaires MSC, citant l'exemple des panneaux ajourés que le père $\mathrm{R}$. Von Peij aurait prétendument poussé les Asmat à sculpter (ce dernier m'a assurée de la carence de l'accusation ${ }^{35}$ ). Elle oriente la confection de l'œuvre dans les limites de ce qu'elle juge acceptable : elle ne présente pas à l'artiste une photo de l'objet fini mais elle le décrit et arrête le sculpteur au besoin tout autant que l'évêque. Lorsqu'elle travaille avec des groupes de femmes, elle leur dit ce qu'il convient de tresser pour assurer les ventes. Il s'agit de « leur fournir seulement quelques idées, leur indiquer la direction mais ne jamais dire que faire, à personne. Pourquoi nos jeunes vontils dans les écoles d'art ? Pour apprendre. Donc c'est ce que nous essayons de donner ». Les Asmat interrogés sur les groupes de travail d'Ursula Konrad expriment tantôt leur indifférence, tantôt leur incompréhension, tantôt leur colère par rapport à leur sensation d'être traités comme des animaux.

Si les Konrad n'eurent pas d'influence affichée sur les Asmat, ils en eurent sur les Croisiers. Par la publicité tapageuse qu'ils firent autour de l'art asmat, certains croisiers interrogés soulignent que les Konrad leur firent prendre conscience de la valeur de l'art et de la culture. Ils les incitèrent à agir de même par leur venue en Asmat, l'organisation d'expositions et la constitution d'une collection entière. Outre leur enthousiasme communicatif pour soutenir l'art asmat, les Konrad représentèrent une aide financière substantielle pour la mission et un encouragement de taille par le don de pans entiers de leurs collections aux Croisiers pour leurs musées, comme nous l'avons vu.

\section{Des contestataires parmi les Croisiers}

Malgré l'engouement généralisé pour l'activité de sculpture, la promotion de l'art asmat telle qu'elle a lieu ne rencontre pas que des partisans. Deux Croisiers, dont je ne citerai pas le nom à leur demande, critiquent sévèrement la manière dont l'évêque Sowada et les Konrad « préservent la culture ». Du côté de l'évêché, l'opinion générale est qu'il n'existe pas d'Asmat suffisamment formé pour assurer la relève. Sans les efforts des Croisiers, il ne subsisterait plus de traces de la culture matérielle pour instruire les petits-enfants des Asmat sur la vie quotidienne de leurs grands-pères. Erik Sarkol, le conservateur du musée (employé par l'évêché), considère qu'il est le seul à pouvoir assumer ce poste, principalement faute de candidats parmi les Asmat ${ }^{36}$. Les missionnaires anonymes ne sont pas de cet avis : ils déplorent que le musée d'Agats et la vente aux enchères fussent toujours pris en charge par le diocèse et que «certaines personnes » ne laissassent pas les Asmat les gérer eux-mêmes. " Il est temps de couper le cordon », dit l'un des deux. Alphonse contredit cette assertion : la vente aux enchères est entre les mains des Asmat et du gouvernement ( $\mathrm{sic}$ ) actuel ${ }^{37}$. Un missionnaire se demande au nom

35. Dans sa maison de St. Joost, il possède un de ces panneaux acquis à son arrivée en 1956; il n'aurait donc pu en être l'instigateur.

36. Jusqu'au début 2004, il était aidé par Boni, un Asmat licencié en anthropologie de l'université d'UnCen à Jayapura. Cependant, Boni le quitta pour embrasser une carrière mieux rémunérée : celle de fonctionnaire.

37. Lors de la dernière vente, en 2001, Alphonse Sowada et Ursula Konrad faisaient partie du comité de sélection des œuvres et de l'organisation générale de la vente. Alphonse assure qu'à présent, le comité de sélection de la vente annuelle d'Agats ne regroupe que des membres asmat du musée et du gouvernement; les Konrad et lui n'ont donc plus rien à voir avec l'organisation de la vente. Sans mettre ces paroles en doute, notons qu'ils participèrent à la vente de 2001 et s'y rendirent à nouveau en 2003. 
de quoi ou de qui l'évêque et les Konrad se permettent de sélectionner des objets asmat et désapprouve totalement ces ventes. D'après lui, les Konrad pousseraient les gens à céder leurs sculptures à un prix dérisoire la plupart du temps. En 1997, il se vit confier par des Asmat leur sentiment de ne pas avoir de contrôle sur le jugement des objets et l'évaluation du « premier prix ».

Par ailleurs, l'évêque se serait plus occupé de recevoir les visiteurs que de culture et se serait laissé influencer par les Konrad dont le missionnaire anonyme dénonce la motivation financière ${ }^{38}$. Dans les premiers temps, beaucoup aurait été réalisé pour la préservation de la culture mais cela aurait changé au début des années 1980. A cet égard, la publication Asmat Drums est devenue Crosier Drums à la même époque, détail révélateur d'un état d'esprit davantage orienté vers les missionnaires que vers les Asmat. Chaque année, l'évêque apparaît lors de la vente aux enchères d'Agats, c'est-à-dire lors d'une activité commerciale où les gens sont photographiés à des fins esthétiques. L'autre Croisier anonyme souligne le peu d'implication de l'évêque dans les paroisses, contrairement aux affirmations de l'intéressé. Absorbé par la gestion du musée, il aurait poussé les Croisiers à encourager la production artistique « depuis son bureau » d'Agats. Il était absent de cette culture qu'il prétendait défendre et préférait les mondanités à la fréquentation de la population.

Dans le même ordre d'idées, un des deux missionnaires regrette que le nouveau musée d'Agats soit régi indépendamment des Asmat. La vente des poteaux d'ancêtres bisj, par exemple, leur ôterait leur caractère sacré. Le missionnaire déplore que des objets soient exposés en dehors des périodes de temps prescrites, comme les masques des esprits qui ne sont pas sensés être exhibés en permanence. Jadis, une pièce annexe au musée d'Agats servait à stocker ce qui ne pouvait être exposé, faute de place. Supprimer cette pièce a en même temps détruit "le mystère » : on sait désormais où les masques demeurent, et ils n'ont plus l'air de surgir de nulle part lors de leur sortie pendant les funérailles ${ }^{39}$. Désappointés par ce déballage, certains Asmat s'en seraient plaints à ce Croisier. Ce dernier aurait tenté d'infléchir les responsables mais « il y a des gens avec qui on ne peut pas parler ». Remarquons qu'Alphonse conteste le bien-fondé de ces déclarations. Il n'y a pas à son avis d'objet que l'on ne puisse montrer. De son côté, le conservateur Erik Sarkol ne nie pas que des visiteurs asmat puissent souffrir d'avoir vu certains objets, mais ils sont minoritaires. Un jour, une famille de la Pantai Kasuari revint au musée pour apaiser l'esprit d'un objet par un rituel : cet esprit avait provoqué la maladie d'un enfant après leur visite du musée.

En parallèle, une catégorie à part entière de la vente aux enchères d'Agats est formée par les story boards, des panneaux sculptés illustrant des mythes dont, toujours à l'avis de ce Croisier anonyme, il ne peut subsister de trace matérielle. Ces panneaux sont apparus à l'instigation des missionnaires et continuent à être produits en raison de la

38. Un bémol paraît utile à ce sujet. Avant de créer sa propre galerie, dont une partie est destinée à la vente, Ursula Konrad joua un rôle dans le Volksmuseum de l'Université de Heidelberg. Ses fonds personnels sont alloués pour une grande part à l'acquisition d'art asmat, et donc que son but soit de s'enrichir prête à critique. Son souci déclaré est de conserver les objets sous forme de collection et, dans la mesure du possible, de les vendre à un musée pour éviter d'inonder le marché.

39. Le porteur du masque incarne réellement le défunt. Pendant la semaine qui suit le décès, il vit avec sa famille où il est nourri et logé, le temps pour les proches de faire leur deuil. 
demande. Prenant peu à peu conscience que l'on ne peut partager les secrets avec des gens d'une autre culture, certains sculpteurs considèrent cette exhibition des secrets comme un sacrilège. Les Asmat sont supposés se rallier progressivement à douze « groupes culturels » (cultural groups). Ces groupes sont identifiés sur la base théorique de l'intercompréhension linguistique, des formes de sculpture et du déroulement des fêtes ${ }^{40}$, selon une classification évolutive qui dépend de la connaissance acquise de « la région asmat» par les collectionneurs. Sur le terrain, certains ignorent encore de quel groupe ils font partie, surtout parmi les femmes. Le Croisier anonyme va jusqu'à qualifier ces agissements de « génocide culturel »...

\section{Un christianisme artistique}

En parallèle aux contestations des uns et des autres, les missionnaires et les collectionneurs d'art tentèrent d'obtenir des Asmat qu'ils leur façonnent des objets personnalisés. Schneebaum (1989 : 55) écrit que les missionnaires, aussi bien néerlandais qu'américains, demandèrent aux sculpteurs de confectionner des crucifix et des statues de Vierge à l'enfant. Le père R. von Peij MSC confirme que les principaux changements culturels dus aux missionnaires apparaissent dans le domaine de l'art. En 1956, il demanda à un homme de lui sculpter un crucifix. Au lieu de la croix escomptée, le résultat fut un arbre stylisé ${ }^{41}$. Renseignements pris, les énormes racines de cette mangrove servaient habituellement de réceptacle à une boule de feuilles dans laquelle sont logés les ossements des morts. Le sculpteur avait donc fait le rapprochement entre la signification mortuaire du crucifix et les pratiques funéraires locales. L'improvisation se raréfia par la suite; à Pirimapun et à Atsj, par exemple, les missionnaires en place prièrent les Asmat de sculpter des crucifix selon des patrons universellement acceptés plutôt que de laisser libre cours à leur imagination.

Aux dires d'Ursula Konrad, l'assemblage des panneaux ajourés de Sawa-Erma en boîtes ou en meubles est né de la suggestion du père van Dongen. Le père von Peij conseilla aux gens d'Atsj de sculpter de petits objets en bois pour vendre aux touristes, généralement peu désireux de transporter les sculptures volumineuses traditionnelles (il était en Asmat de 1956 à 1962). La production artistique dans son ensemble - et non spécifiquement d'inspiration chrétienne - augmente notamment à cause des prêtres, qui commandent des œuvres aux sculpteurs qui leur paraissent les plus compétents. Clarence, un des Croisiers, pense que l'apparition d'éléments chrétiens dans la sculpture est surtout due répondre à des fins mercantiles. Dave, un autre Croisier, confirme que le passage du représentatif et du symbolique au réaliste non traditionnel marque le caractère majeur du changement dans l'art asmat, et que les missionnaires y ont joué un rôle important. Cependant, dit Dave, « sans les missions, ils auraient perdu leur art ». Par contre, Dave a peine à évaluer le rôle exact des missionnaires dans le changement : il se demande s'ils déterminèrent la nouvelle direction prise par l'art ou s'ils le laissèrent se développer dans la direction qu'il tendait à prendre spontanément.

40. A examiner les textes de plus près, les critères de définition sont esthétiques plutôt que linguistiques : les distinctions s'opèrent d'après les mythes, les fêtes, les chants, l'art et les objets rituels (Konrad \& Winkelmann, $1996: 303$ ).

41. L'allure de ce "crucifix » caractéristique est un entrelacs végétal allongé reposant sur une base plate et surmonté à droite d'une tête de profil regardant à gauche. 


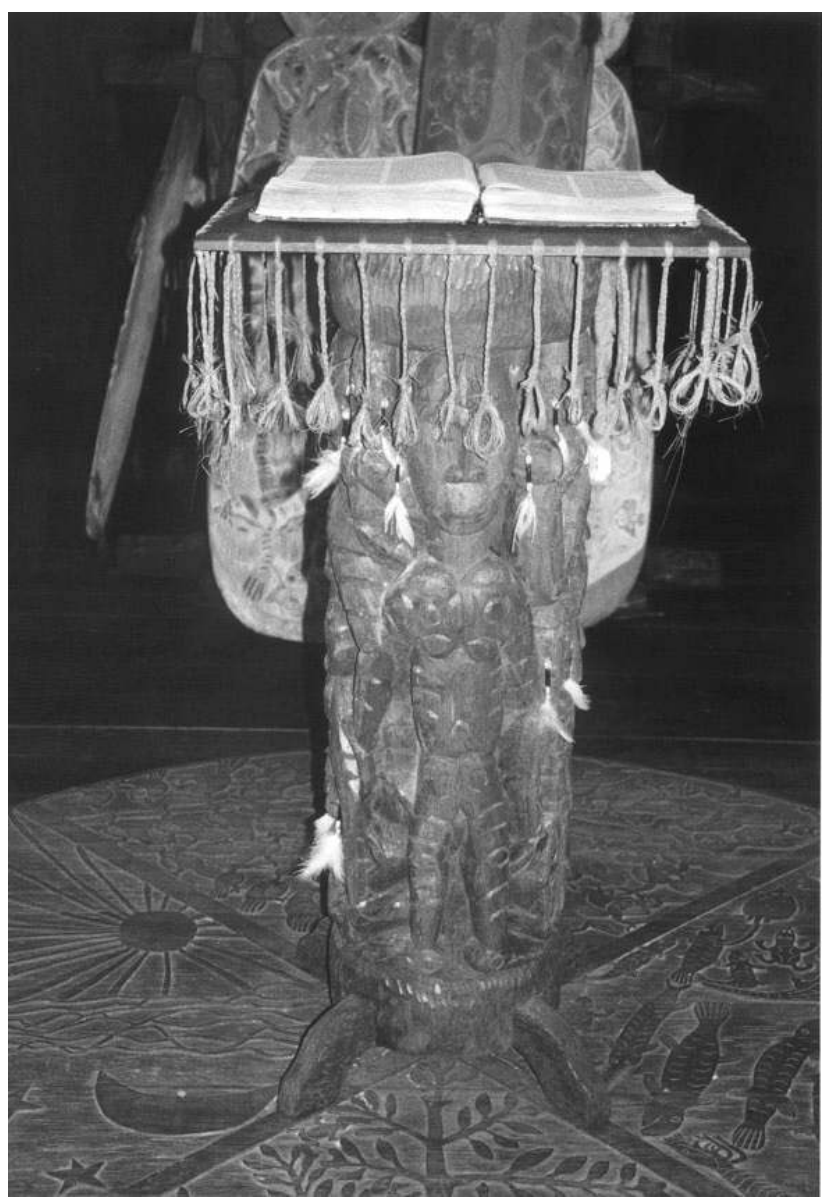

Lutrin en bois de fer dans la nouvelle église de Sawa, inaugurée en février 2004 (C) photo AdH 2004).

Une autre évolution notée par le Croisier Virgil concerne les personnages sculptés sur les poteaux d'ancêtres bisj. Au départ réservés aux morts au combat, les bisj voient apparaître « une écolière ou son père décédé de malaria ». Virgil se félicite de la persistance des cérémonies des bisj, même s'ils ne sont plus destinés à pourrir dans les champs de sagou comme jadis ${ }^{42}$ et si la moitié est fabriquée pour la vente. Sur les grands bisj de la cathédrale d'Agats, on reconnaît le père Tillemans, l'archevêque de Merauke à l'arrivée des Croisiers en 1958, et le père Jan Smit, assassiné par un fonctionnaire en 1965. Aux dires de Virgil, les bisj d'Agats se composent donc d'un mélange de guerriers morts à la guerre, de vieux chefs de guerre de Sjuru, des quatre évangélistes et de catéchistes ${ }^{43}$. Cela

42. Selon Konrad et Winkelmann (1996 : 304), une sculpture assume une fonction spécifique dans une fête particulière; une fois la fête terminée, elle perd toute valeur. L'abandon des mâts dans les champs de sagou n'aurait donc pas de connotation rituelle et ne viserait qu'à jeter les sculptures après usage.

43. Des catéchistes mimika (la région mimika se situe au nord-ouest de l'Asmat) furent envoyés dans certains villages asmat par les pères du Sacré-Cœur, précédant ainsi les missionnaires. 
semble être le seul cas de missionnaires représentés sur les poteaux d'ancêtres. Alphonse prétend que Jésus fait également partie des ancêtres des Asmat, même s'il est absent des bisj. Jim, un autre Croisier, pense que les missionnaires ne font pas partie de l'existence des Asmat parce qu'ils déménagent sans cesse et ne restent pas en permanence avec eux. Aucun Croisier ne mourut en territoire asmat sauf le père Smit, ce qui explique peut-être sa présence sur les bisj d'Agats.

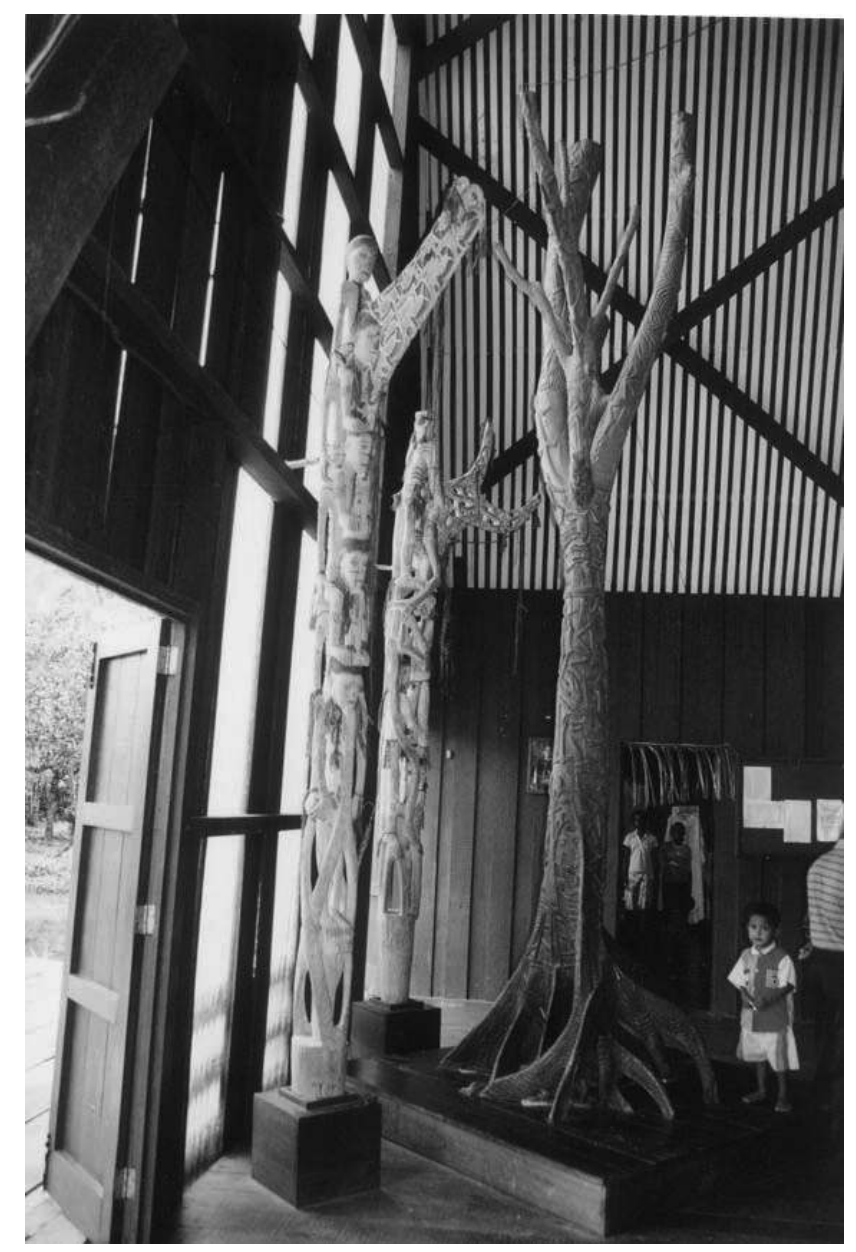

Poteaux d'ancêtres bisj et « arbre de vie » dans la cathédrale d'Agats (C photo AdH 2004).

\section{Le Christ d'Atsj}

Comme le crucifix-mangrove du père von Peij, le Christ en croix d'Atsj comporte une singularité qui étonne au premier coup d'œil : il a les poignets liés au-dessus de la tête. Le prêtre javanais d'Atsj m'expliqua en 2001 que le supplice de la crucifixion n'existant pas chez les Asmat, ceux-ci n'auraient pu comprendre que le Christ meure de cette façon. De son propre chef, l'artiste asmat avait jugé plus judicieux de le représenter 
de cette manière suggestive. Malgré tout, des empreintes de mains gravées aux extrémités gauche et droite de la croix rappellent le châtiment initial. On trouve ces empreintes sur la plupart des crucifix portables fabriqués actuellement, quelle que soit la position du Christ, acquérant de la sorte un sens autonome. La nature de l'entrave des mains et des pieds pose également question. Un examen rapide révèle des maillons, écartant l'hypothèse d'une corde : le sculpteur semble plutôt avoir cherché à représenter une chaîne ou des menottes. S'agit-il d'une allusion aux emprisonnements de guerriers asmat par les gouvernements néerlandais puis indonésien dans le cadre de la lutte contre la chasse aux têtes ? Je n'ai pas pu retrouver le sculpteur pour préciser ses intentions. Au pied du Christ d'Atsj sont également sculptés deux femmes en pagne assises et en prières et un chien debout, la gueule ouverte. En matérialisant des personnages qui déambulent habituellement sur les routes-passerelles, l'artiste a sans doute voulu intégrer le Christ à la vie quotidienne, donc se l'approprier, et c'est justement ce que cherchent à faire les Croisiers quand ils parlent d'inculturation.

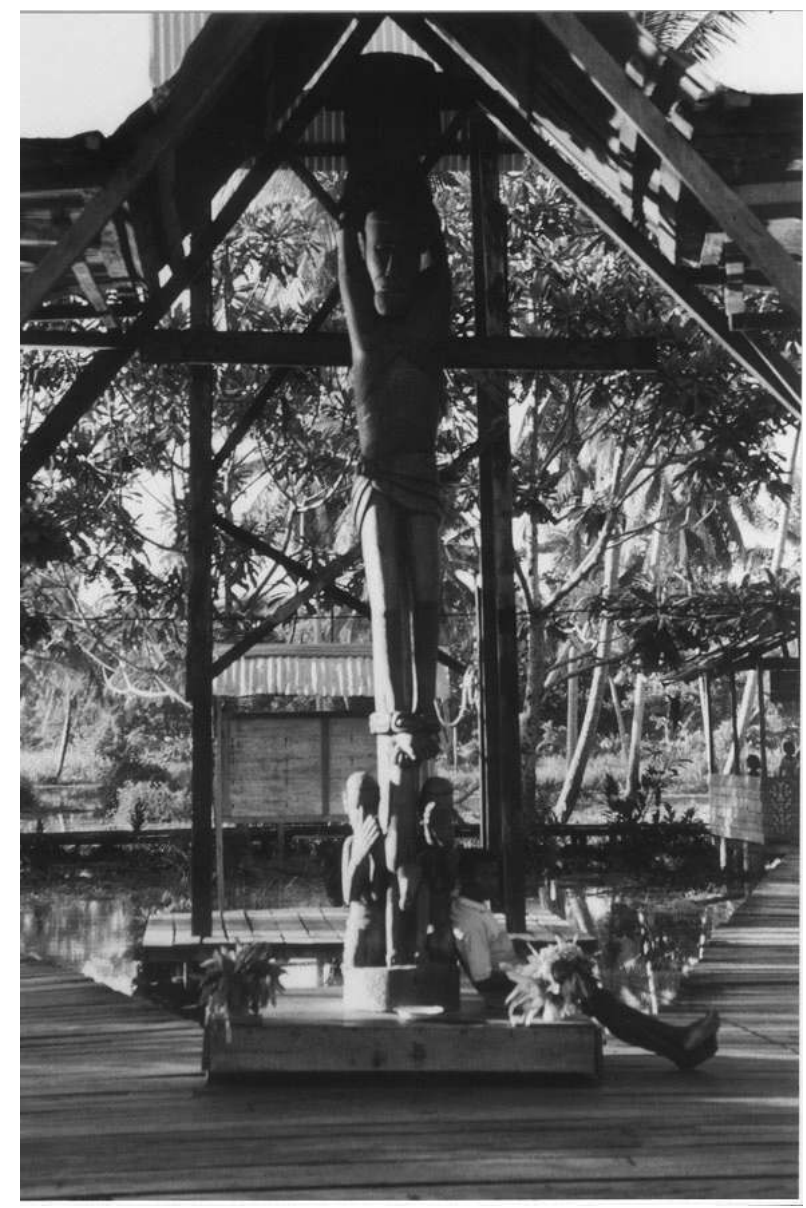

Les mains du Christ d'Atsj sont nouées au-dessus de la tête; à ses pieds, deux femmes en prières et un chien aboyant (C photo AdH 2004). 
Cette sculpture illustre de façon frappante la notion de pensée métisse de Gruzinski (1999 : 39-40). En effet, il fut demandé au sculpteur de réaliser un Christ crucifié, vraisemblablement sur base d'un modèle. Dans la copie, la croix et le Christ sont bien présents, mais d'autres éléments autochtones, la «déviation » du modèle copié, apparaissent, et pas seulement dans la décoration annexe au personnage principal mais dans le principe même de la crucifixion, qui donne une impression d'éléments à première vue discordants.

\section{Pour ou contre l'influence occidentale}

Que penser de tous ces mélanges? Au sujet des influences occidentales, conscientes ou inconscientes, les avis sont partagés. Tom, le provincial des Croisiers américains, reconnaît que « le monde devient une communauté plus large »; il s'en est aperçu pendant qu'il négociait des sculptures avec les Asmat en tant que directeur du musée d'art asmat à Shoreview. Au départ, il pensait comme certains puristes qu'il ne fallait ni promouvoir, ni exposer ni parler de l'art ou de la culture d'après l'arrivée des missionnaires. Puis il constata que le monde des Asmat n'était plus celui des jours anciens. Les motifs des boucliers, des poteaux d'ancêtres bisj et des tambours sculptés à cette époque provenaient de rituels et de pratiques qui n'ont plus cours, comme la chasse aux têtes. La société se développa et s'exprima différemment d'auparavant, au gré des arrivées d'Occidentaux en Asmat.

Schneebaum (1989 : 51-5) montre moins de tolérance. Dans l'ensemble, il considère l'influence étrangère dommageable à la qualité de l'art asmat sur le plan des idées (éclosion de nouveaux thèmes ${ }^{44}$ ) et de la technique (ciseau en métal et clou au lieu de la hache en pierre et du ciseau en os de casoar ${ }^{45}$ ). L'utilisation d'outils en métal ajouta de la finesse et de la complexité aux sculptures, augmenta la taille des bisj et favorisa l'apparition de nouvelles formes. Alphonse Sowada désapprouve Schneebaum quand il déplore la mort de l'art asmat et qu'il qualifie les nouvelles créations de « rebut pour aéroport » (airport junk). Remarquons que ceux qui incriminent «les étrangers» se comportent parfois comme eux. John, un des Croisiers interviewés, déclare que lorsque Schneebaum collecta des objets pour le musée d'Agats, il le pria de demander aux Asmat d'organiser une fête de la « pirogue des âmes » (uramum) à Jamasj, à Yufri, à As-Atat et à Kapi, et ce dans l'intention d'acheter la pirogue à la fin de la fête. Il commanda aussi une sculpture $u m u$ dans la région de Jamasj. D’après John, il voulait surtout acquérir des sculptures pour son bénéfice personnel.

\section{Conclusions}

Au début ou presque de la christianisation des basses terres asmat, la sculpture asmat fait l'objet de manifestations promotionnelles qui l'éloignent de plus en plus de la société dont elle est issue. Des artistes européens y trouvent une source d'inspiration inépuisable,

44. Le casoar, le cochon, l'avion, la figure humaine mise en mouvement (et non plus figée), plus petite que la figure d'ancêtre, mais aussi le dragon Naga vu sur une boîte d'allumettes et l'aigle Garuda d'un poster, deux créatures mythiques que les Asmat ont intégrées dans leur corpus de mythes, remplaçant les personnages d'origine.

45. Oiseau coureur (Ratites) faisant partie comme l'autruche de l'ordre des struthioniformes dont on consomme la chair et dont on utilise les plumes pour décorer les parures et les sculptures. 
au gré des collectes d'objets quasi-annuelles des collectionneurs. De cette manière, l'art asmat sert de stimulant à des courants nouveaux d'art contemporain, connus en Europe et aux Etats-Unis grâce aux efforts de collectionneurs motivés et de leurs relations. De plus, les membres de ce réseau international puisent dans la production asmat ce qu'ils considèrent comme représentatif d'une tradition éteinte (la chasse aux têtes) pour en faire le commerce. Et leur vision essentialiste de la société asmat concourt à en vendre l'image à des prix appréciables.

En attendant, les sculpteurs aussi ont envie de reconnaissance. Plusieurs représentants de la tradition me firent part de leur souci d'éviter la dilapidation de certains objets et d'obtenir pour d'autres un prix calculé selon les mécanismes du " commerce équitable ». Une institution fut créée en 2000 en partie dans cet objectif : la LMAA (ou Lembaga Musyawarah Adat Asmat), le Centre Consultatif Traditionnel Asmat. Ce projet naquit à l'instigation des trois Asmat les plus impliqués dans la politique indonésienne: Ernest Nditsjim, Nikolaus Ndepi et Yufen Biakai. Les membres de la lembaga sont des représentants de la tradition wair'ipitsj de tout l'Asmat à l'exception de son président, Yufen Biakai. Yufen, élu pour ses relations avec l'extérieur et sa maîtrise des langues étrangères, est d'ailleurs tellement critiqué qu'il y a lieu de douter quant à la prolongation de son mandat. Les représentants de la tradition des douze " groupes culturels » asmat (rumpun, que l'on peut traduire par «famille ») composent autant d'entités appelées Far, ou Forum Adat Rumpun. Chaque Far est présidé par un président (ketua) et un vice-président (wakil), à la façon des chefs de parti indonésiens. Soucieux de fonder une institution traditionnelle asmat, les membres refusèrent l'acte notarié du gouvernement ${ }^{46}$, qui en aurait fait une institution indonésienne. Le souci d'autochtonie n'empêche pourtant pas la lembaga de définir ses organes et ses membres par des termes indonésiens - donc non asmat - et de se priver de la reconnaissance légale qu'aurait impliqué la constitution d'un acte notarié. Enfin, la «tradition » ne semble pas plus préservée qu'avant. Les membres se réunissent moins d'une fois l'an, et le seul changement visible est l'estampille imprimée en relief sur le courrier des ketua.

Du point de vue des missionnaires, plusieurs années furent nécessaires aux pères croisiers avant d'adopter un comportement de collectionneurs, et à la sculpture asmat avant de trouver sa place dans le marché international de l'art. Les missionnaires furent les initiateurs de mécanismes commerciaux et d'initiatives promotionnelles dans lesquels leur influence décrut au fil du temps, d'autres prenant le relais. Les événements organisés autour de la sculpture asmat et de son acquisition en Occident finirent parfois par échapper à leur contrôle. « L'art asmat » se modifia au gré des rencontres, parfois sciemment, et pas toujours par la volonté missionnaire, et inspira de nouvelles formes d'art contemporain. Finalement, l'art asmat ne fait pas vivre que les sculpteurs dans le marais : il est un puissant incitant à la création pour une série d'artistes auxquels les missionnaires servent de référence. En effet, les missionnaires ont fortement contribué - et contribuent encore - à faire la publicité de l'art asmat en prenant la parole lors de ventes et de mondanités. Ils en viennent à jouer un rôle de faire-valoir dans un réseau d'art contemporain qui les dépasse et dont peu d'Asmat soupçonnent l'existence. Après avoir lutté pour faire reconnaître la sculpture asmat comme un art à part entière, les missionnaires promeuvent la sculpture allemande, peut-être à leur insu. La remise en contexte des objets vendus par

46. Il semble que ce type de « centre traditionnel » soit répandu dans d'autres sociétés indonésiennes. 
des « missionnaires anthropologues » ajoute du crédit à leur description comme œuvres d'art, et les aventures racontées par ces pionniers achèvent de les entourer d'une aura exotique d'exception. Les sculpteurs sont des chasseurs de têtes sous la responsabilité des missionnaires, qui parlent en leur nom. En quelque sorte, acquérir un objet fait participer l'acheteur à la domestication de la société asmat par la culture occidentale; par la même occasion, l'insertion de l'art asmat dans le marché de l'art équivaut à insérer la société asmat dans la société mondiale. Les Asmat se rendent-ils compte de ces manœuvres qui semblent les concerner au premier chef? Pas vraiment, mais suffisamment toutefois pour justifier la fondation d'une lembaga. Les efforts des missionnaires auront au moins contribué à cette prise de conscience. En outre, si les Asmat visitent peu le musée d'Agats, beaucoup clament leur enthousiasme par rapport à la vente aux enchères comme source de revenus.

On aurait envie de se demander si à force d'avoir cherché à mettre la culture à l'avantplan, l'évangélisation proprement dite est passée au second. La réalité est plus complexe. Sans sauver d'abord la culture, selon Alphonse, les missionnaires ne pouvaient pas « faire grand-chose » au niveau de la religion. Une base culturelle était nécessaire comme « base de foi » sur laquelle les missionnaires pouvaient construire la religion et sans laquelle les Asmat n'auraient jamais compris la Bible. Il en résulte que les bancs des églises sont clairsemés sauf en présence d'un dignitaire ou d'une fête : cinquante ans après l'arrivée des Croisiers, pas un d'entre eux n'affirme que les Asmat sont convertis. D'après Alphonse, «c'est une question de patience ». Il semble être le seul à le penser.

\section{Remerciements}

Cet article n'aurait pas pu être mené à bien sans la relecture attentive de Bernard Sellato et d'Athanase Pandiris, que je remercie. Merci aussi à Florence Brunois pour ses commentaires avisés. 


\section{Références bibliographiques}

Appadurai, A., 1996. Modernity at Large. Cultural Dimensions of Globalization. Minneapolis \& London: University of Minnesota Press.

BeCKer, A. L., 2000. Crosiers Remembered : USA 1854-1999. Onamia : Crosier Press.

Gruzinski, S., 1999. La pensée métisse. Paris : Fayard.

Helfrich, K., 1996. « The Asmat », in Asmat. Myths and Rituals. The Inspiration of Art, G. \& U. Konrad (eds.), pp. 35-43. Venise : Pizzi Amilcare/Erizzo Editrice.

Hontheim, (de) A., 2005. « Après cinquante ans de christianisation, les Asmat sont-ils convertis ? L'inculturation vue par les pères croisiers» (article réalisé dans le cadre du colloque «Inculcation, adaptation, inculturation » tenu à Marseille les 19 et 20 mars 2004) (à paraître).

Hoogerbrugge, J., 1993. «Art Today : Woodcarving in Transition », in Asmat Art. Woodcarvings of Southwest New Guinea, D.A.M. Smidt (ed.), pp. 150-153. New York: George Braziller. Leiden : Rijksmuseum voor Volkenkunde.

KLEIN, L. A., 1989. «Introduction », in People of the River, People of the Tree : Change and Continuity in Sepik and Asmat, Minnesota Museum of Art (ed.), pp. 1-4. Saint Paul : Minnesota Museum of Art.

Konrad, G. \& U. and C. Winkelmann, 1996. «Asmat Art», in Asmat. Myths and Rituals. The Inspiration of Art, G. \& U. Konrad (eds.), pp. 303-436. Venise : Pizzi Amilcare/Erizzo Editrice.

Minnesota Museum of Art (ed.), 1989. People of the River, People of the Tree : Change and Continuity in Sepik and Asmat. Saint Paul : Minnesota Museum of Art.

Mischke, B., 1957. Lettre du 20 octobre au master general F. M. Rees (Source : archives des Croisiers à Shoreview).

O’Reilly, P., 1958. « Musées et expositions missionnaires. Musées missionnaires et ethnologiques », in Histoire universelle des missions catholiques. L'Eglise catholique face au monde non chrétien, S. Delacroix (dir.), pp. 59-70. Paris : Librairie Grund

ReEs, F. M., 1957. Lettre du 8 octobre au provincial Benno Mischke. Amersfoort (Source : archives des Croisiers à Shoreview).

SCHNEEBAumt, T.,

1989. " Change in Asmat Art», in People of the River, People of the Tre $e$ : Change and Continuity in Sepik and Asmat, Minnesota Museum of Art (ed.), 51-57. Saint Paul : Minnesota Museum of Art.

1990. Embodied Spirits. Ritual Carvings of the Asmat. Salem : Peabody Museum of Salem.

1991. La demeure des esprits. Traduit de l'américain par A. Labeau. Paris : Actes Sud.

Trenkenschun, F., 1982. " Some Additional Notes on Zegwaard from a 1970 Vantage », in An Asmat Sketch Book $n^{\circ}$ 1, F. A. Trenkenschuh (ed.), pp. 31-8. Agats : The Asmat Museum of Culture and Progress.

Van Arsdale, P. W., 1978. An Asmat Sketch Book $n^{\circ} 5$ : Perspectives on Development in Asmat. Agats : The Asmat Museum of Culture and Progress.

VAn DER ZEe, P., 1996. Etsjopok: Avenging the Ancestors. The Asmat Bisj Poles and a Proposal for a Morphological Method. Working Papers in Ethnic Art 8. Ghent : University of Ghent, Department of Ethnic Art.

Zegwadrd, G. A., 1959. «Headhunting practices of the Asmat of Netherlands New Guinea », American Anthropologist 61 (6), pp. 1020-1041.

Zegwatrd, G. \& J. BoelaArs, 1982. «An Annotated Translation of 'De Sociale Structuur van de Asmatbevolking' », in An Asmat Sketch Book $n^{\circ}$ 1, F. A. Trenkenschuh (ed.), pp. 13-29. Traduit du néerlandais et annoté par F. Trenkenschuh et J. Hoggerbrugge. Agats : The Asmat Museum of Culture and Progress. 
Astrid De Hontheim

\section{Filmographie}

Corillion, J.-M., 2001. Asmat. Zed, Odyssée/Rai 3, Canal +, RTBF/TV5.

NeEdles, C., 1993. A World Away. WCCO News.

Schneebaumt, T., 2000. Keep the River on Your Right. A Modern Cannibal Tale. Wave Films, Lifer Films Production, Stolen Cars Production. 